\title{
Synthesis of the First Rare Earth Metal Bis(alkyl)s Bearing Indenyl Functionalized N-Heterocyclic Carbene
}

Baoli Wang, ${ }^{1,2}$ Dun Wang, ${ }^{1,2}$ Dongmei Cui, ${ }^{* 1}$ Wei Gao, ${ }^{1}$ Tao Tang, ${ }^{1}$ Xuesi Chen, ${ }^{1}$ Xiabin Jing ${ }^{1}$

\section{Experiment section}

Figure S1. X-ray structure of 2 with $40 \%$ probability of thermal ellipsoids. Hydrogen atoms are omitted for clarity.

Figure S2. X-ray structure of $\mathbf{3}$ with $40 \%$ probability of thermal ellipsoids. Hydrogen atoms are omitted for clarity.

Figure S3. ${ }^{1} \mathrm{H}$ NMR spectrum of (IndH-NHC-H)Br.

Figure S4. ${ }^{13} \mathrm{C}$ NMR spectrum of (IndH-NHC-H)Br.

Figure S5. ${ }^{1} \mathrm{H}-{ }^{13} \mathrm{C}$ HMQC spectrum of (IndH-NHC-H)Br.

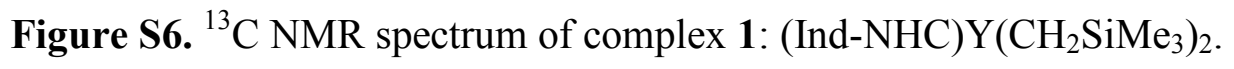

Figure S7. ${ }^{1} \mathrm{H}$ NMR spectrum of complex 2: (Ind-NHC) $\mathrm{Lu}\left(\mathrm{CH}_{2} \mathrm{SiMe}_{3}\right)_{2}$.

Figure S8. ${ }^{13} \mathrm{C}$ NMR spectrum of complex 2: (Ind-NHC) $\mathrm{Lu}\left(\mathrm{CH}_{2} \mathrm{SiMe}_{3}\right)_{2}$.

Figure S9. ${ }^{1} \mathrm{H}-{ }^{13} \mathrm{C}$ HMQC spectrum of complex 2: (Ind-NHC) $\mathrm{Lu}\left(\mathrm{CH}_{2} \mathrm{SiMe}_{3}\right)_{2}$.

Figure S10. ${ }^{1} \mathrm{H}$ NMR spectrum of complex 3: (Ind-NHC)Sc $\left(\mathrm{CH}_{2} \mathrm{SiMe}_{3}\right)_{2}$.

Figure $\mathrm{S11}{ }^{13} \mathrm{C}$ NMR spectrum of complex 3: (Ind-NHC) $\mathrm{Sc}\left(\mathrm{CH}_{2} \mathrm{SiMe}_{3}\right)_{2}$.

Figure S12. ${ }^{1} \mathrm{H}$ NMR spectrum of polyisoprene. 
Stable 1. Crystal data and structure refinement for $\mathbf{1}$.

Stables 2-4 are crystallographic parameters and bond lengths and angles for $\mathbf{1}$.

Stable 5. Crystal data and structure refinement for 2.

Stables 6-8 are crystallographic parameters and bond lengths and angles for $\mathbf{2}$.

Stable 9. Crystal data and structure refinement for 3.

Stables 10-12 are crystallographic parameters and bond lengths and angles for 3 . 


\section{Synthesis of $\mathrm{Y}\left(\mathrm{CH}_{2} \mathrm{SiMe}_{3}\right)_{4} \mathrm{Li}(\mathrm{THF})_{4}$}

(a) To a THF (40 mL) suspension of $\mathrm{YCl}_{3}(1.547 \mathrm{~g}, 7.92 \mathrm{mmol})$ was added dropwise a THF solution $(5 \mathrm{~mL})$ of $\mathrm{LiCH}_{2} \mathrm{SiMe}_{3}(2.983 \mathrm{~g}, 31.68 \mathrm{mmol})$ at room temperature under vigorous stirring $(30 \mathrm{~min})$. The reaction mixture became clear gradually and maintained for $30 \mathrm{~min}$. Then, volatiles were driven off to give grayish white solids, which were extracted with benzene $(20 \mathrm{~mL})$ to give two-layer liquors and solids. Filtered off solids and separated benzene from the oily layer of the liquors. The oil was thoroughly dried up by vacuum to afford white powders of $\mathrm{Y}\left(\mathrm{CH}_{2} \mathrm{SiMe}_{3}\right)_{4} \mathrm{Li}(\mathrm{THF})_{4}(4.325 \mathrm{~g}, 74.5 \%)$.

(b) $\mathrm{LiCH}_{2} \mathrm{SiMe}_{3}(0.157 \mathrm{~g}, 1.67 \mathrm{mmol})$ in $5 \mathrm{~mL}$ THF was added dropwise to $\mathrm{Y}\left(\mathrm{CH}_{2} \mathrm{SiMe}_{3}\right)_{3}(\mathrm{THF})_{2}(0.826 \mathrm{~g}, 1.67 \mathrm{mmol})$ in $5 \mathrm{~mL}$ THF with vigorous stirring. After addition, the volatiles were removed in vacuum and the resulted white solids were washed with hexane $(20 \mathrm{~mL} \times 3)$ and dried in vacuum to give $\mathrm{Y}\left(\mathrm{CH}_{2} \mathrm{SiMe}_{3}\right)_{4} \mathrm{Li}(\mathrm{THF})_{4}$ in a yield of $85.3 \%$ (1.044 g). ${ }^{1} \mathrm{H}$ NMR (400 MHz, $\mathrm{C}_{6} \mathrm{D}_{6}, 25$ ${ }^{\circ} \mathrm{C}$ ): $\delta-0.31$ (br s, $8 \mathrm{H}, \mathrm{CH}_{2} \mathrm{SiMe}_{3}$ ), 0.54 (s, 36H, $\mathrm{CH}_{2} \mathrm{SiMe}_{3}$ ), 1.45 (br s, $16 \mathrm{H}, \mathrm{THF}$ ),

3.67 (br s, 16H, THF). Anal. Calcd for $\mathrm{C}_{32} \mathrm{H}_{76} \mathrm{LiO}_{4} \mathrm{Si}_{4} \mathrm{Y}$ (\%): C, 52.42; H, 10.45 . Found: C, 52.64; H, 9.89.

\section{Synthesis of $\mathrm{Lu}\left(\mathrm{CH}_{2} \mathrm{SiMe}_{3}\right)_{4} \mathrm{Li}(\mathrm{THF})_{4}$}

Following the same procedure described previously for the synthesis of $\mathrm{Y}\left(\mathrm{CH}_{2} \mathrm{SiMe}_{3}\right)_{4} \mathrm{Li}(\mathrm{THF})_{4}, \mathrm{LuCl}_{3}(1.517 \mathrm{~g}, 5.39 \mathrm{mmol})$ reacted with $\mathrm{LiCH}_{2} \mathrm{SiMe}_{3}$ (2.031 g, $21.57 \mathrm{mmol})$ to afford $3.101 \mathrm{~g}$ of $\mathrm{Lu}\left(\mathrm{CH}_{2} \mathrm{SiMe}_{3}\right)_{4} \mathrm{Li}(\mathrm{THF})_{4}(70.2 \%) .{ }^{1} \mathrm{H}$ NMR (400 MHz, $\left.\mathrm{C}_{6} \mathrm{D}_{6}, 25^{\circ} \mathrm{C}\right): \delta-0.38\left(\mathrm{~s}, 8 \mathrm{H}, \mathrm{CH}_{2} \mathrm{SiMe}_{3}\right), 0.59$ (s, 36H, $\mathrm{CH}_{2} \mathrm{SiMe}_{3}$ ), 
1.41-1.48 (m, 16H, THF), 3.57(br s, 16H, THF). Anal. Calcd for $\mathrm{C}_{32} \mathrm{H}_{76} \mathrm{LiLuO}_{4} \mathrm{Si}_{4}$

(\%): C, 46.92; H, 9.35. Found: C, 47.04; H, 8.69.

Synthesis of $\mathrm{Sc}\left(\mathrm{CH}_{2} \mathrm{SiMe}_{3}\right)_{4} \mathrm{Li}(\mathrm{THF})_{4}$

Following the same procedure described previously for the synthesis of $\mathrm{Y}\left(\mathrm{CH}_{2} \mathrm{SiMe}_{3}\right)_{4} \mathrm{Li}(\mathrm{THF})_{4}, \mathrm{ScCl}_{3}(0.822 \mathrm{~g}, 5.43 \mathrm{mmol})$ reacted with $\mathrm{LiCH}_{2} \mathrm{SiMe}_{3}(2.046$ $\mathrm{g}, 21.73 \mathrm{mmol})$ to afford $2.565 \mathrm{~g}$ of $\mathrm{Sc}\left(\mathrm{CH}_{2} \mathrm{SiMe}_{3}\right)_{4} \mathrm{Li}(\mathrm{THF})_{4}(68.5 \%)$. The ${ }^{1} \mathrm{H}$ NMR $\left(400 \mathrm{MHz}, \mathrm{C}_{6} \mathrm{D}_{6}, 25{ }^{\circ} \mathrm{C}\right): \delta 0.27\left(\mathrm{~s}, 8 \mathrm{H}, \mathrm{CH}_{2} \mathrm{SiMe}_{3}\right), 0.60\left(\mathrm{~s}, 36 \mathrm{H}, \mathrm{CH}_{2} \mathrm{SiMe}_{3}\right)$, 1.48-1.53 (m, 16H, THF), 3.53-3.56 (m, 16H, THF). Anal. Calcd for $\mathrm{C}_{32} \mathrm{H}_{76} \mathrm{LiO}_{4} \mathrm{ScSi}_{4}(\%):$ C, 55.77; H, 11.12. Found: C, 56.49; H, 10.33. 


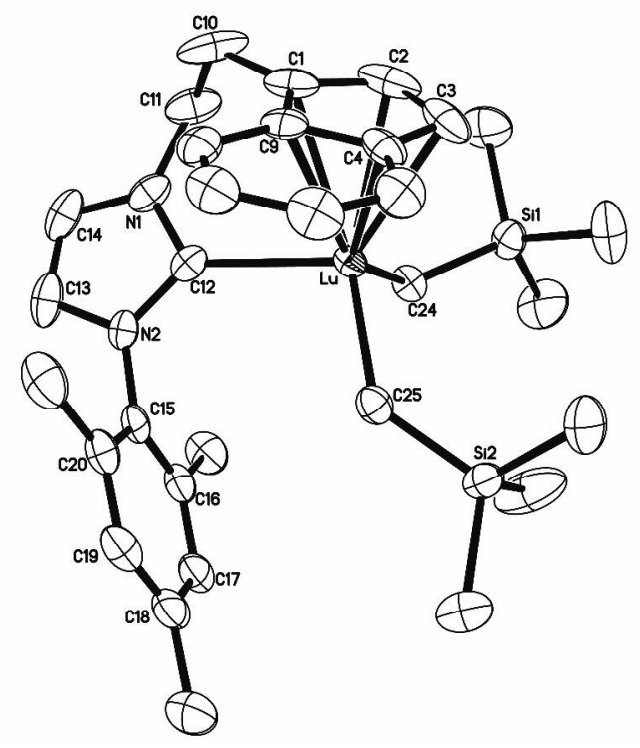

Figure S1. X-ray structure of 2 with $40 \%$ probability of thermal ellipsoids. Hydrogen atoms are omitted for clarity.

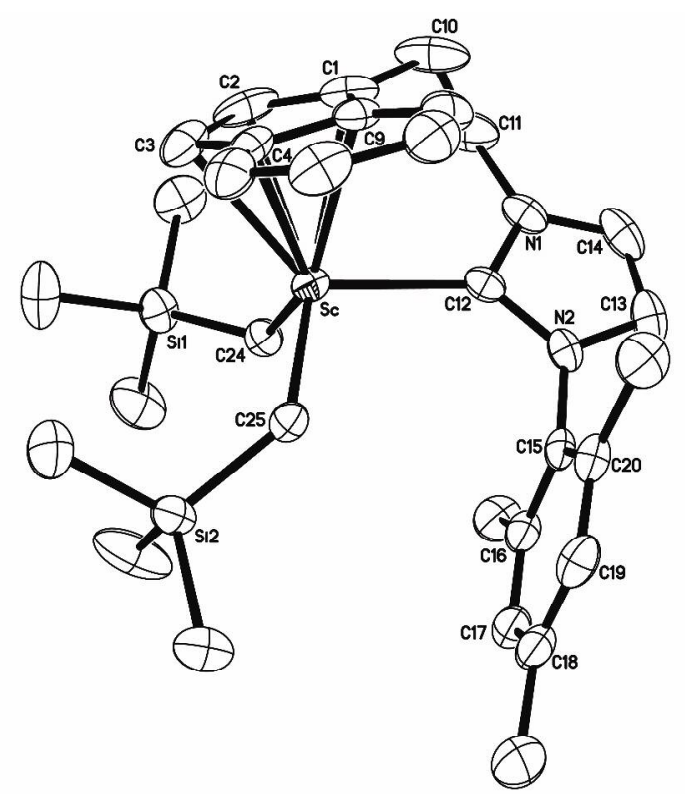

Figure S2. X-ray structure of $\mathbf{3}$ with $40 \%$ probability of thermal ellipsoids. Hydrogen atoms are omitted for clarity. 


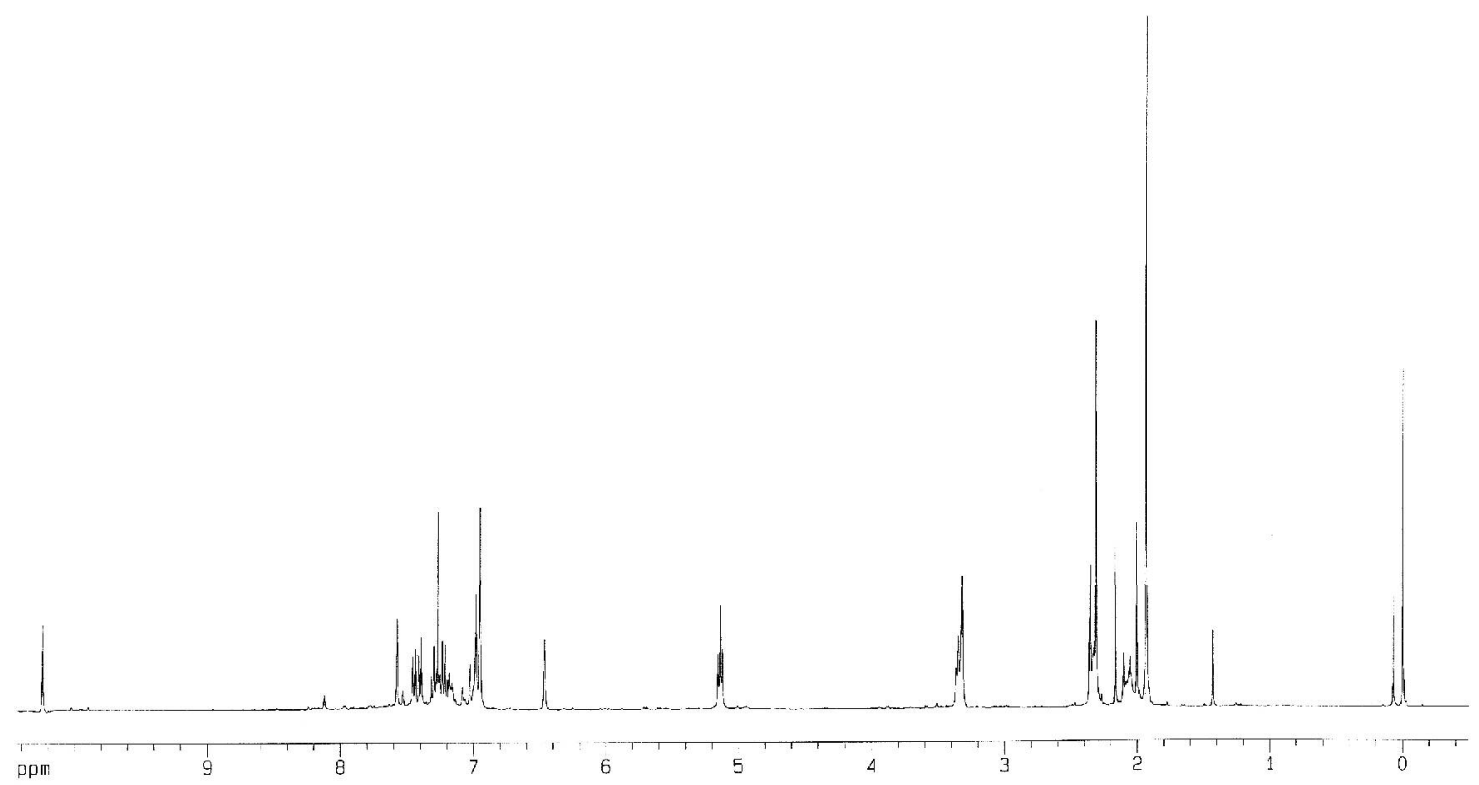

Figure S3. ${ }^{1} \mathrm{H}$ NMR spectrum of (IndH-NHC-H)Br.

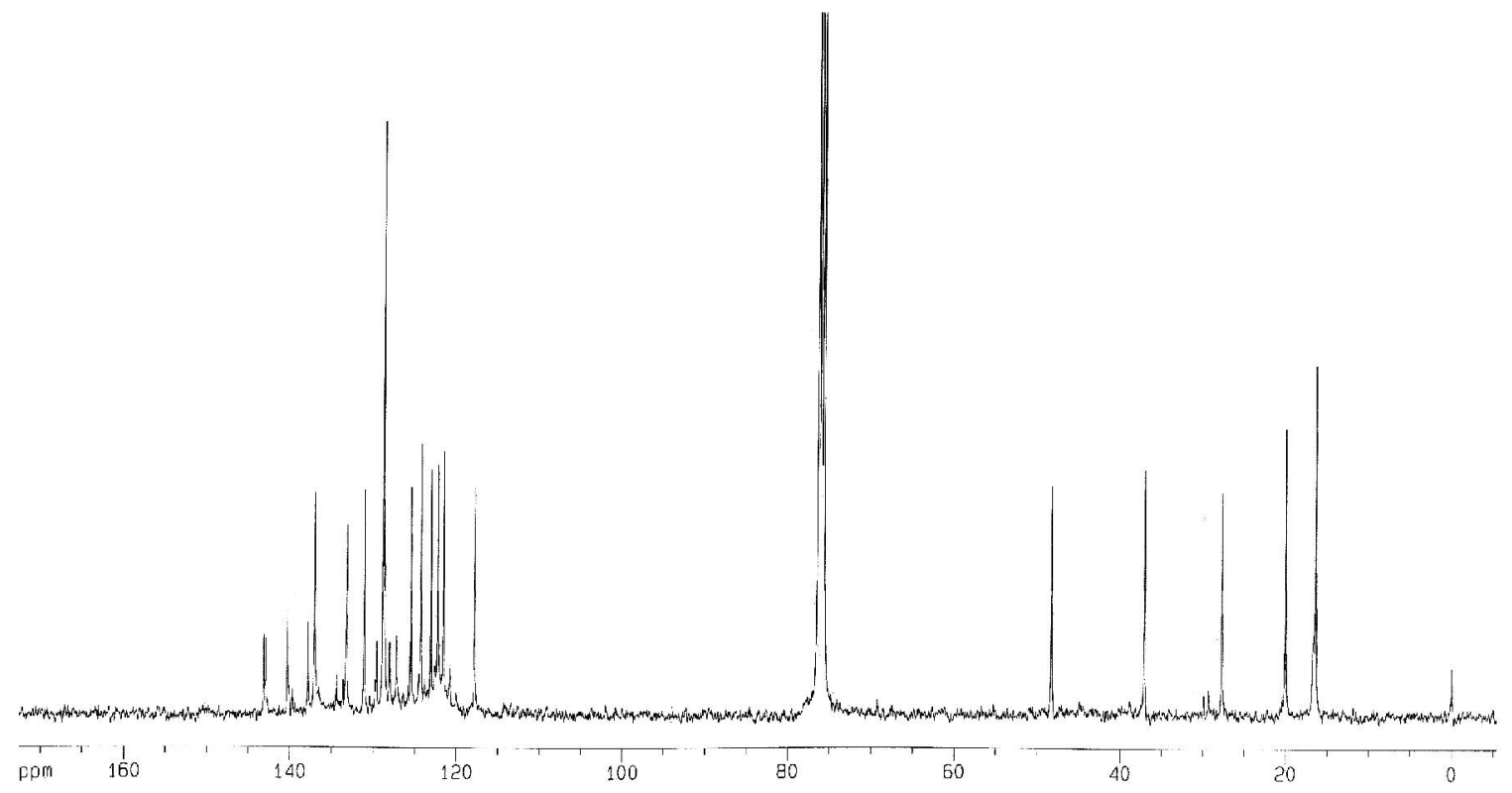

Figure S4. ${ }^{13} \mathrm{C}$ NMR spectrum of (IndH-NHC-H)Br. 


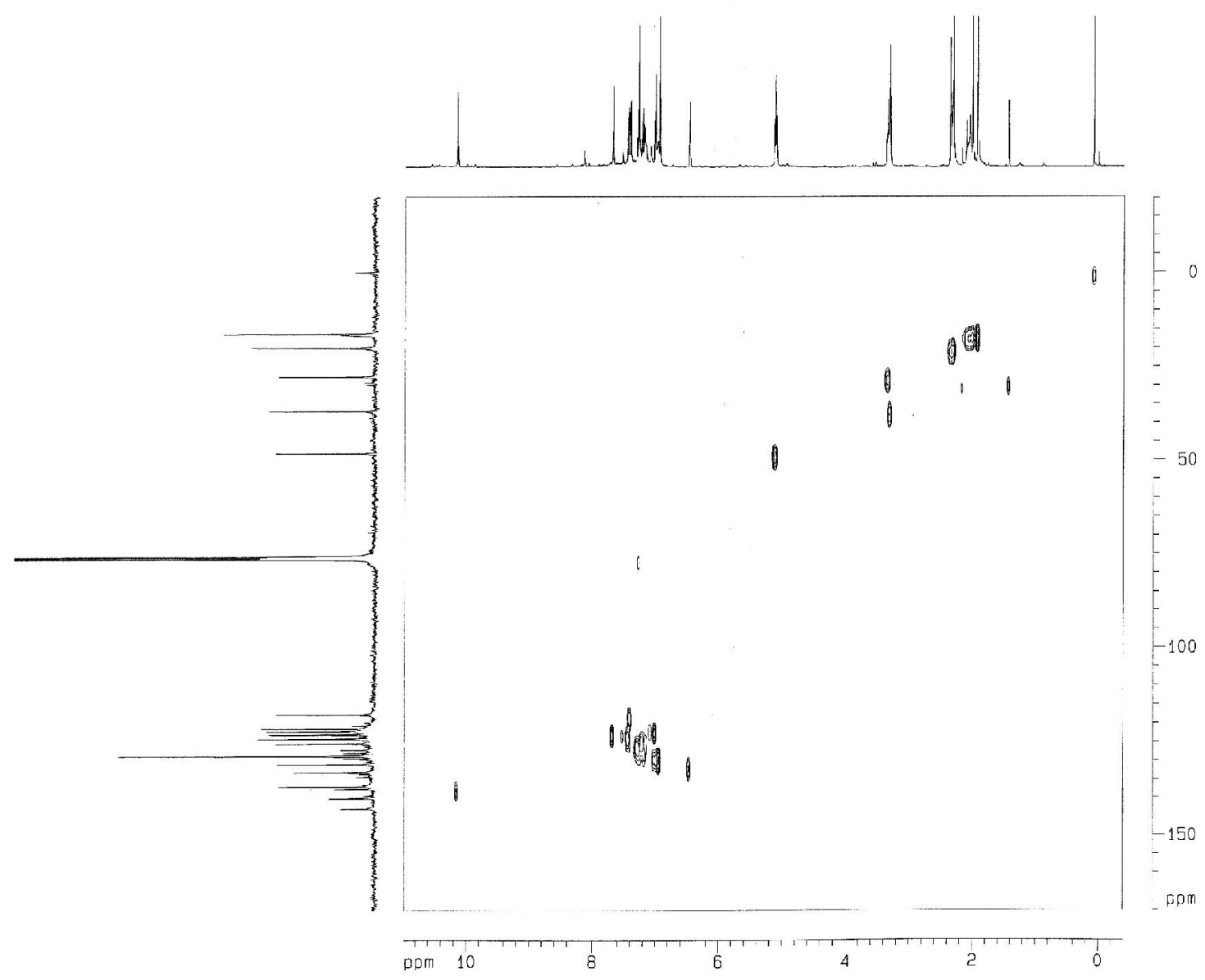

Figure S5. ${ }^{1} \mathrm{H}^{13}{ }^{13} \mathrm{C}$ HMQC spectrum of (IndH-NHC-H)Br 


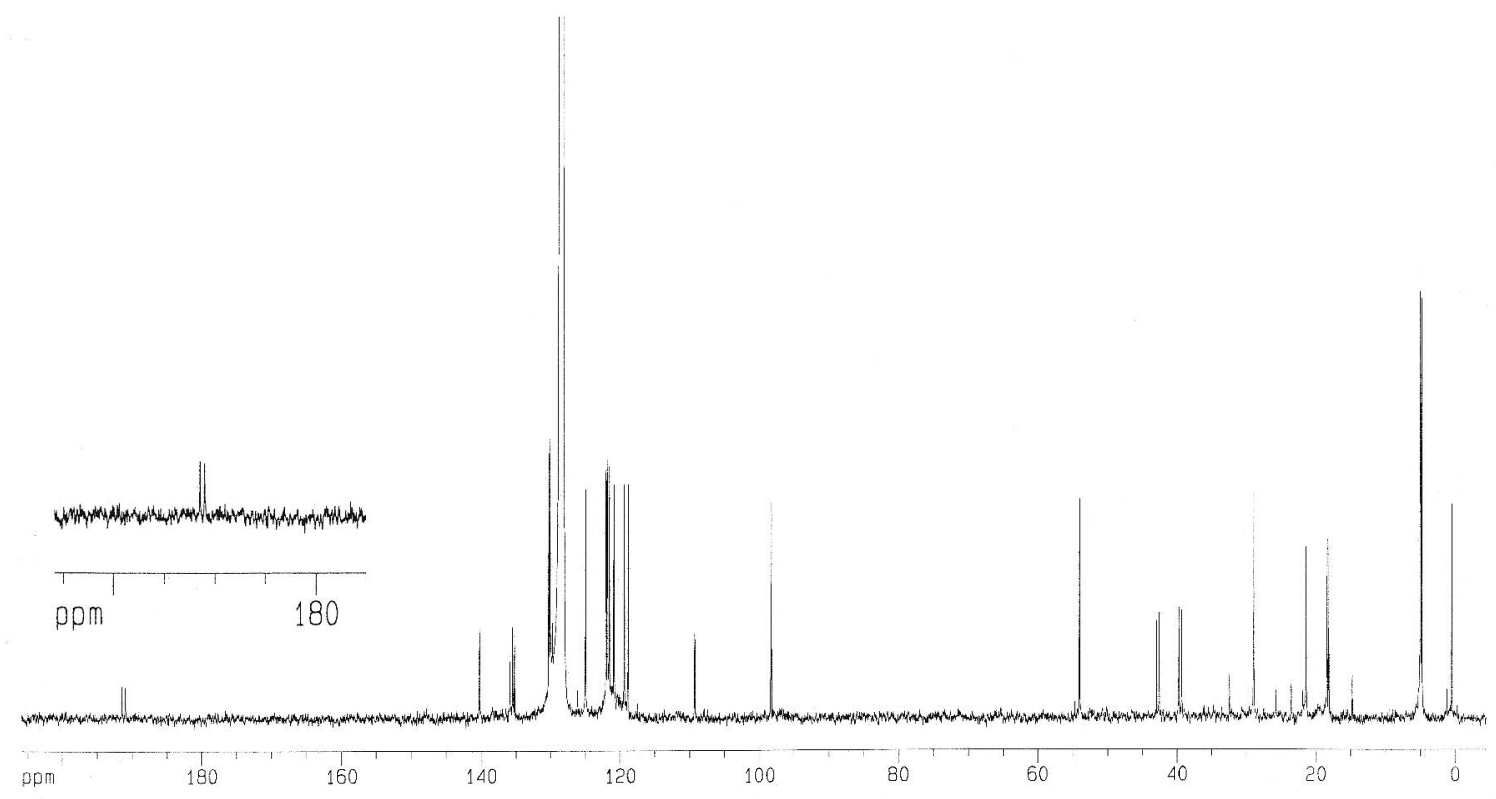

Figure S6. ${ }^{13} \mathrm{C}$ NMR spectrum of complex 1: (Ind-NHC)Y $\left(\mathrm{CH}_{2} \mathrm{SiMe}_{3}\right)_{2}$.

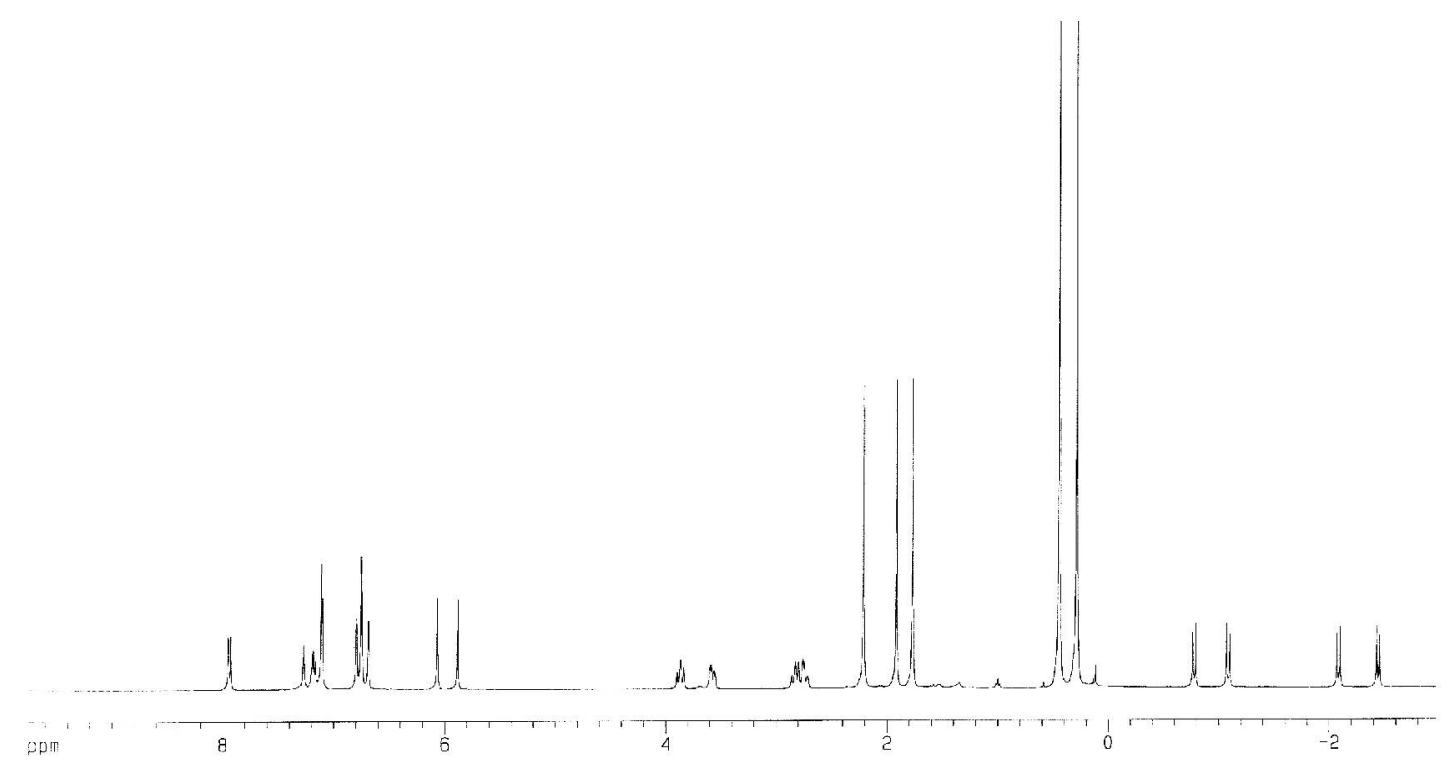

Figure S7. ${ }^{1} \mathrm{H}$ NMR spectrum of complex 2:(Ind-NHC) $\mathrm{Lu}\left(\mathrm{CH}_{2} \mathrm{SiMe}_{3}\right)_{2}$ 


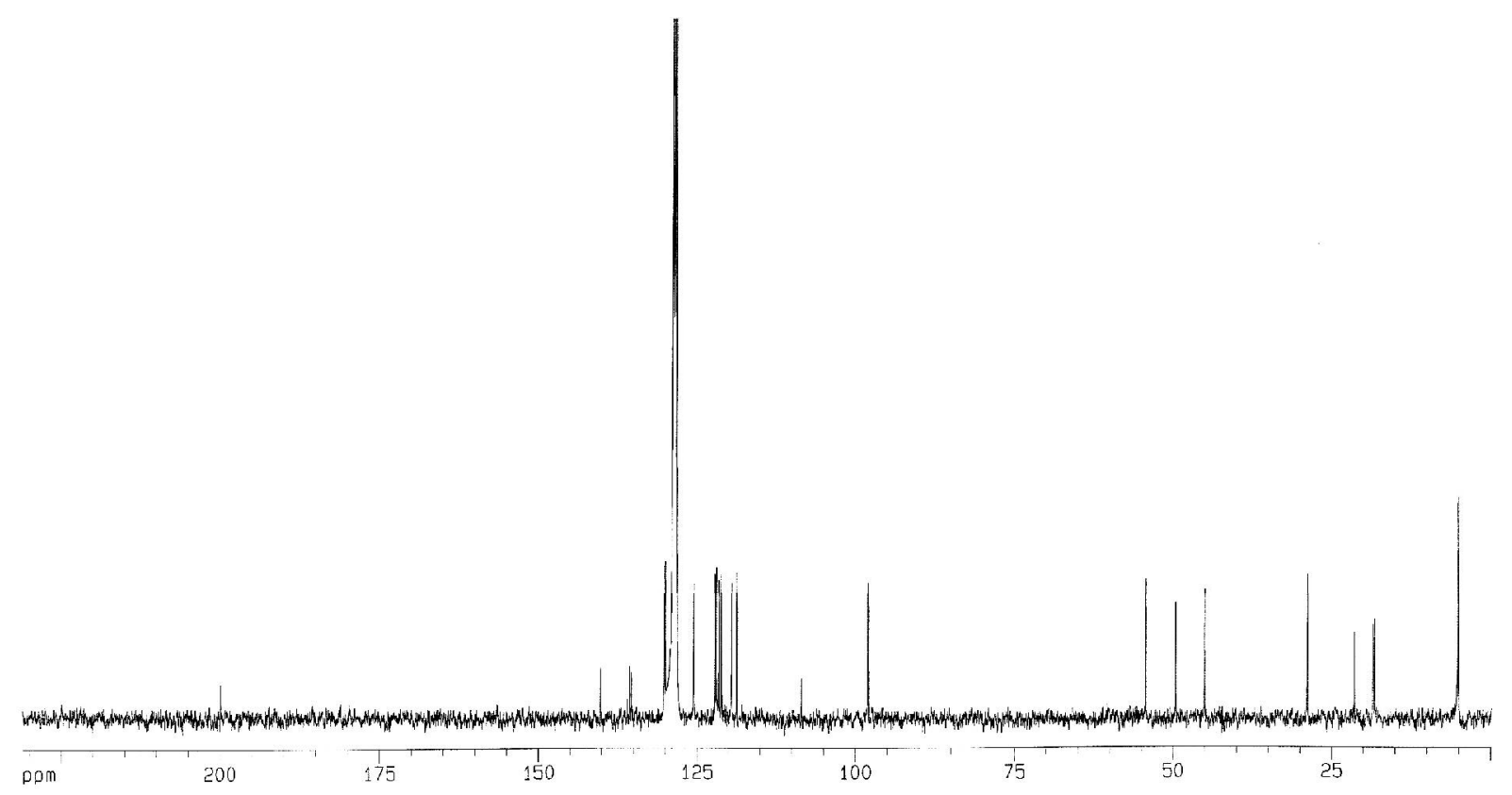

Figure S8. ${ }^{13} \mathrm{C}$ NMR spectrum of complex 2: (Ind-NHC) $\mathrm{Lu}\left(\mathrm{CH}_{2} \mathrm{SiMe}_{3}\right)_{2}$

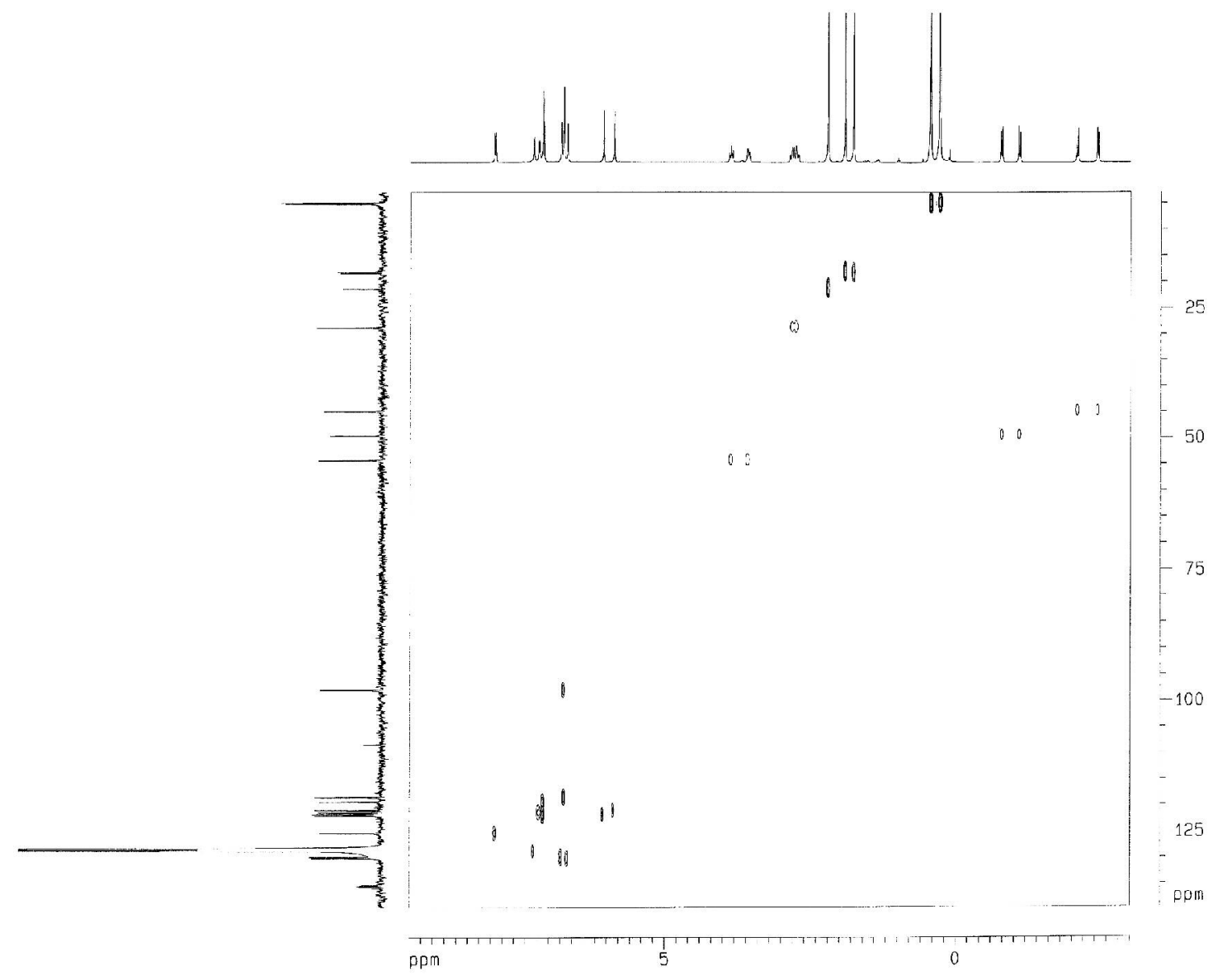

Figure S9. $\quad{ }^{1} \mathrm{H}-{ }^{13} \mathrm{C} \quad \mathrm{HMQC}$ spectrum of complex 3: (Ind-NHC) $\mathrm{Lu}\left(\mathrm{CH}_{2} \mathrm{SiMe}_{3}\right)_{2}$ 


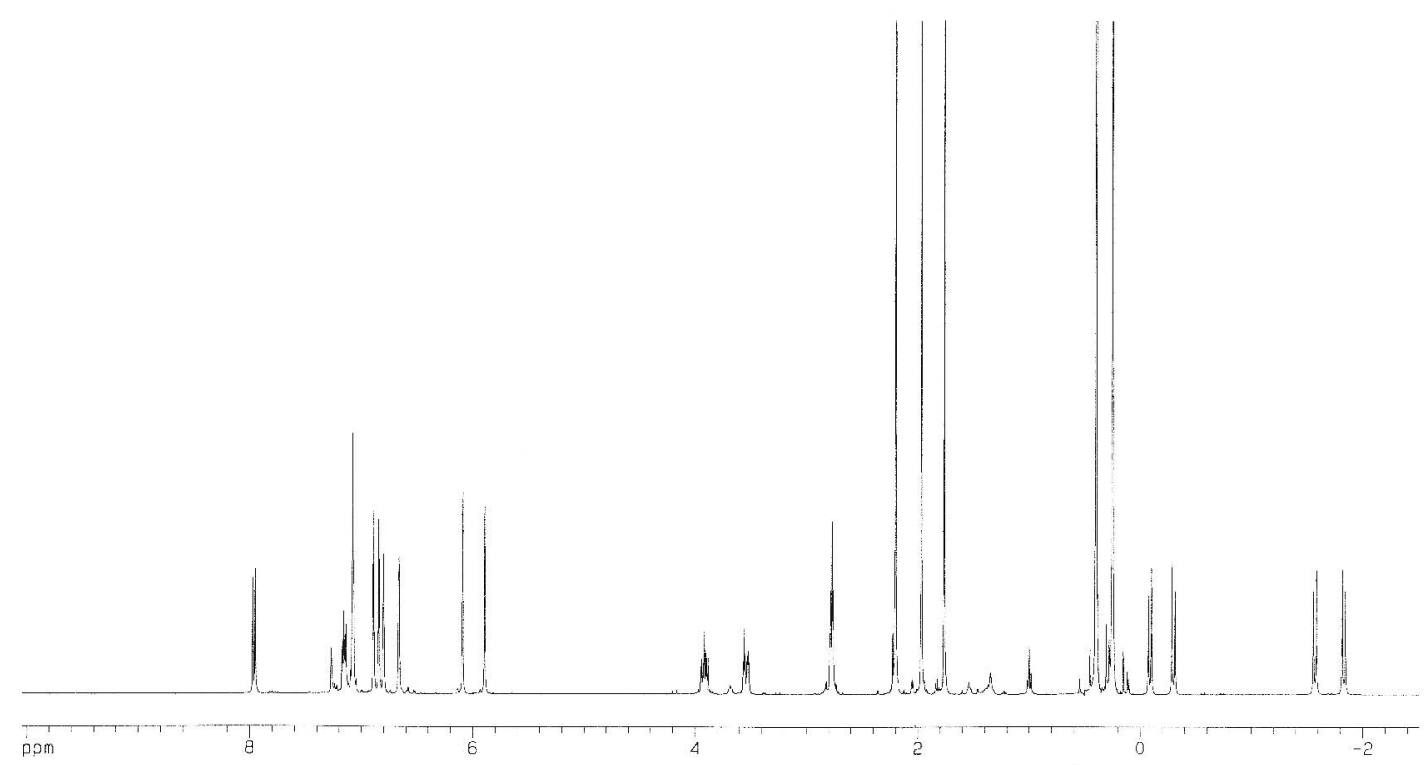

Figure S10. ${ }^{1} \mathrm{H}$ NMR spectrum of complex 3:(Ind-NHC)Sc$\left(\mathrm{CH}_{2} \mathrm{SiMe}_{3}\right)_{2}$.

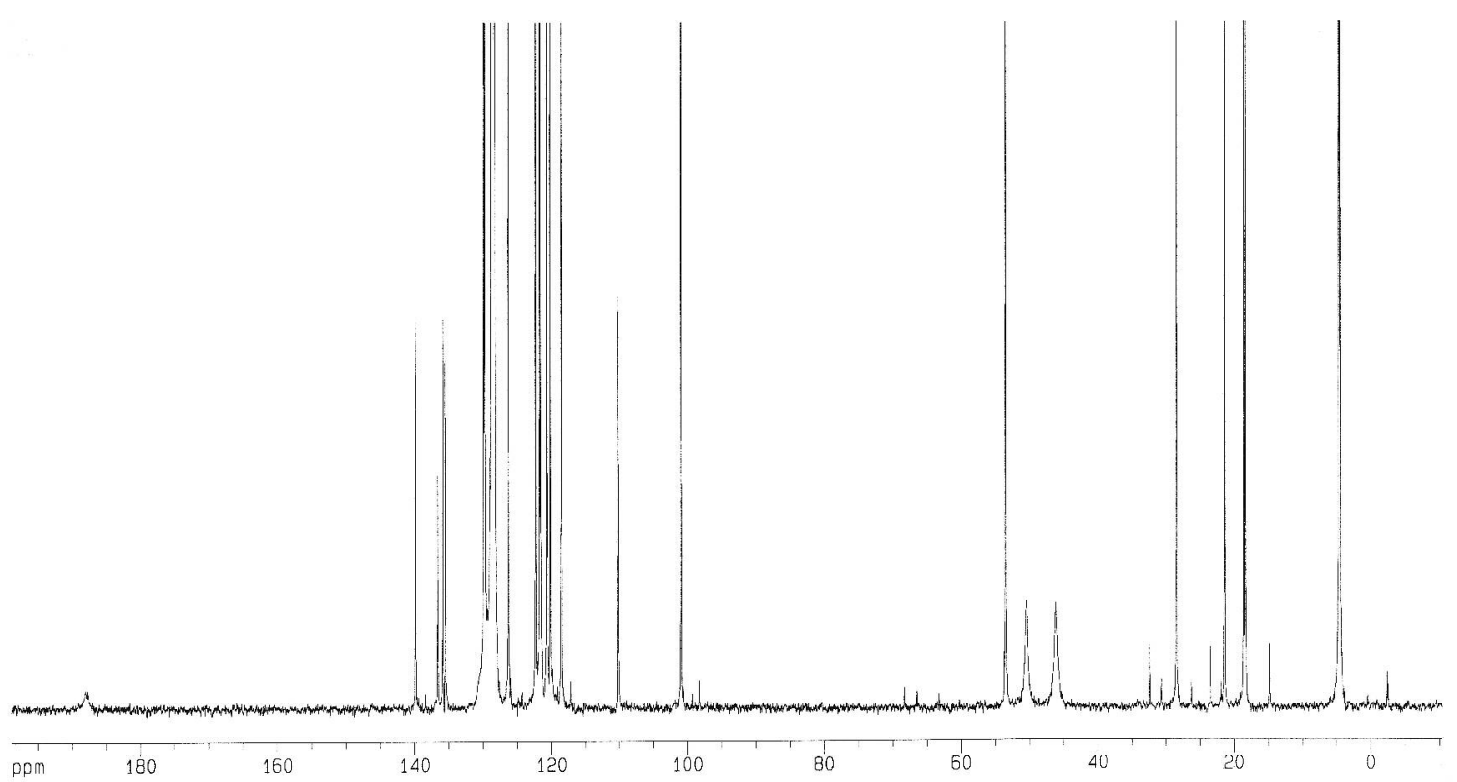

Figure S11. ${ }^{13} \mathrm{C}$ NMR spectrum of complex 3:(Ind-NHC)Sc $\left(\mathrm{CH}_{2} \mathrm{SiMe}_{3}\right)_{2}$. 


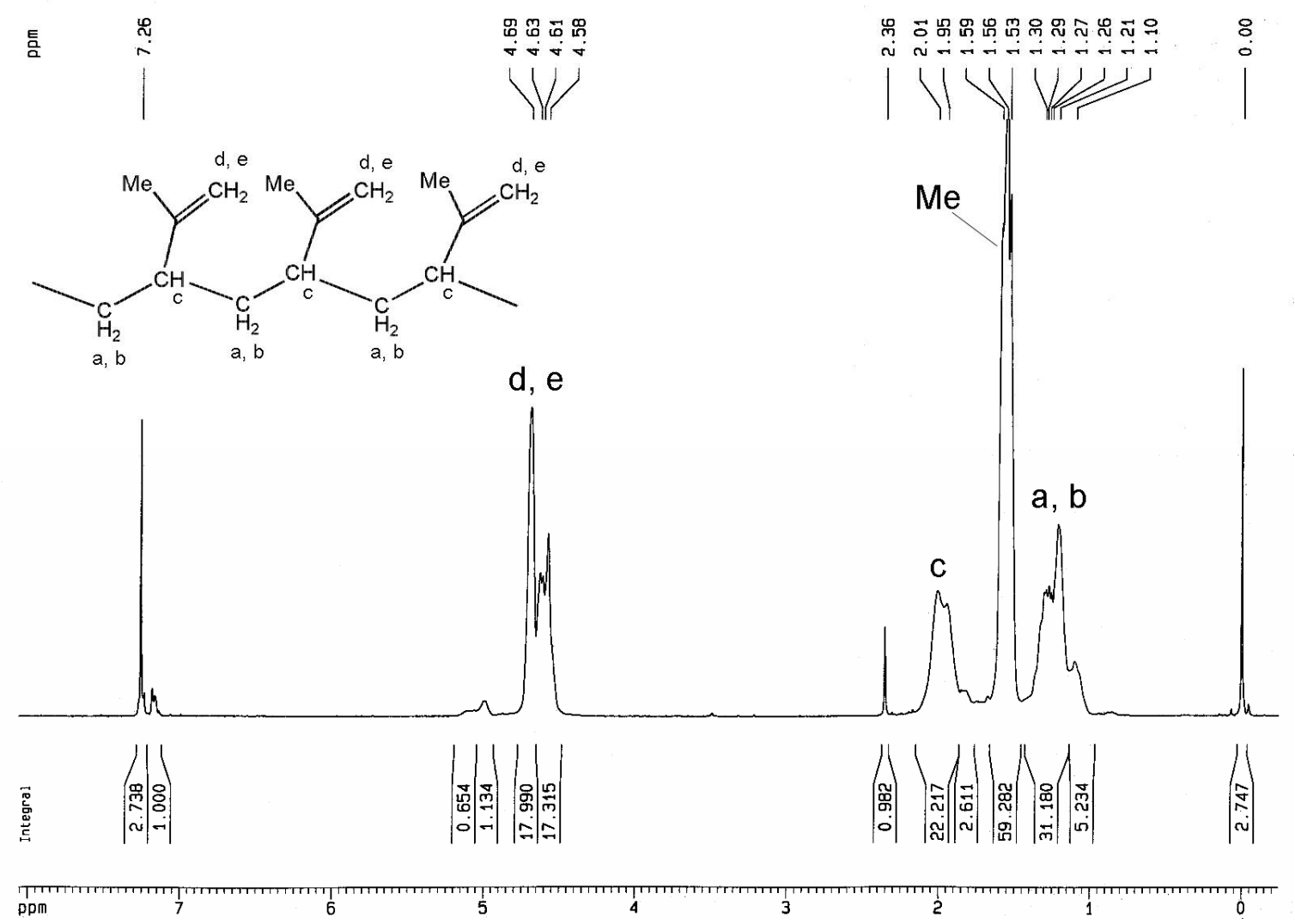

Figure S12. ${ }^{1} \mathrm{H}$ NMR spectrum of polyisoprene 
Stable 1. Crystal data and structure refinement for 1.

\begin{tabular}{|c|c|c|}
\hline Identification code & \multicolumn{2}{|l|}{$\mathrm{r} 071 \mathrm{~m}$} \\
\hline Empirical formula & \multicolumn{2}{|l|}{ C31 H45 N2 Si2 Y } \\
\hline Formula weight & \multicolumn{2}{|l|}{590.78} \\
\hline Temperature & \multicolumn{2}{|l|}{$187(2) \mathrm{K}$} \\
\hline Wavelength & \multicolumn{2}{|l|}{$0.71073 \AA$} \\
\hline Crystal system & \multicolumn{2}{|l|}{ Monoclinic } \\
\hline Space group & \multicolumn{2}{|l|}{$\mathrm{P} 2_{1} / \mathrm{c}$} \\
\hline \multirow{3}{*}{ Unit cell dimensions } & $\mathrm{a}=10.5665(7) \AA$ & $\alpha=90^{\circ}$ \\
\hline & $\mathrm{b}=23.2486(16) \AA$ & $\beta=107.2620(10)^{\circ}$ \\
\hline & $\mathrm{c}=13.9158(10) \AA$ & $\gamma=90^{\circ}$ \\
\hline Volume & \multicolumn{2}{|l|}{$3264.5(4) \AA^{3}$} \\
\hline $\mathrm{Z}$ & \multicolumn{2}{|l|}{4} \\
\hline Density (calculated) & \multicolumn{2}{|l|}{$1.202 \mathrm{Mg} / \mathrm{m}^{3}$} \\
\hline Absorption coefficient & \multicolumn{2}{|l|}{$1.880 \mathrm{~mm}^{-1}$} \\
\hline $\mathrm{F}(000)$ & \multicolumn{2}{|c|}{1248} \\
\hline Crystal size & \multicolumn{2}{|c|}{$0.37 \times 0.24 \times 0.13 \mathrm{~mm}^{3}$} \\
\hline Theta range for data collection & \multicolumn{2}{|c|}{1.75 to $26.04^{\circ}$} \\
\hline Index ranges & \multicolumn{2}{|c|}{$-13<=\mathrm{h}<=12,-22<=\mathrm{k}<=28,-17<=1<=16$} \\
\hline Reflections collected & \multicolumn{2}{|c|}{18226} \\
\hline Independent reflections & \multicolumn{2}{|c|}{$6430[\mathrm{R}(\mathrm{int})=0.0303]$} \\
\hline Completeness to theta $=27.05^{\circ}$ & \multicolumn{2}{|c|}{$99.7 \%$} \\
\hline Absorption correction & \multicolumn{2}{|c|}{ Semi-empirical from equivalents } \\
\hline Max. and min. transmission & \multicolumn{2}{|c|}{0.7854 and 0.5414} \\
\hline Refinement method & \multicolumn{2}{|c|}{ Full-matrix least-squares on $\mathrm{F}^{2}$} \\
\hline Data / restraints / parameters & \multicolumn{2}{|c|}{$6430 / 0 / 334$} \\
\hline Goodness-of-fit on $\mathrm{F}^{2}$ & \multicolumn{2}{|c|}{1.026} \\
\hline Final $R$ indices $[I>2 \operatorname{sigma}(I)]$ & \multicolumn{2}{|c|}{$\mathrm{R} 1=0.0381, \mathrm{wR} 2=0.0878$} \\
\hline $\mathrm{R}$ indices (all data) & \multicolumn{2}{|c|}{$\mathrm{R} 1=0.0604, \mathrm{wR} 2=0.0970$} \\
\hline Largest diff. peak and hole & \multicolumn{2}{|c|}{0.338 and $-0.217 \mathrm{e} \AA^{-3}$} \\
\hline
\end{tabular}


Stable 2. Atomic coordinates ( $x \mathbf{1 0}^{4}$ ) and equivalent isotropic displacement parameters $\left(\AA^{2} x\right.$ $10^{3}$ ) for $1 . \mathrm{U}(\mathrm{eq})$ is defined as one third of the trace of the orthogonalized $\mathrm{U}^{\mathrm{ij}}$ tensor.

\begin{tabular}{|c|c|c|c|c|}
\hline & $\mathrm{x}$ & $\mathrm{y}$ & $\mathrm{Z}$ & $\mathrm{U}(\mathrm{eq})$ \\
\hline $\mathrm{Y}$ & $3428(1)$ & $3414(1)$ & $2884(1)$ & $35(1)$ \\
\hline $\mathrm{N}(1)$ & $5700(2)$ & $3214(1)$ & $1554(2)$ & $48(1)$ \\
\hline $\mathrm{N}(2)$ & $4940(2)$ & $4060(1)$ & $1194(2)$ & $41(1)$ \\
\hline $\operatorname{Si}(1)$ & $868(1)$ & $2246(1)$ & 1891(1) & $50(1)$ \\
\hline $\operatorname{Si}(2)$ & 791(1) & $4434(1)$ & $3197(1)$ & $50(1)$ \\
\hline $\mathrm{C}(1)$ & $5707(3)$ & $2835(1)$ & $3750(2)$ & $56(1)$ \\
\hline $\mathrm{C}(2)$ & $4690(4)$ & $2511(1)$ & $3926(3)$ & $74(1)$ \\
\hline $\mathrm{C}(3)$ & $4105(4)$ & $2811(2)$ & $4568(2)$ & $67(1)$ \\
\hline $\mathrm{C}(4)$ & $4813(3)$ & $3337(1)$ & $4840(2)$ & $53(1)$ \\
\hline$C(5)$ & $4702(3)$ & $3810(2)$ & $5471(2)$ & $63(1)$ \\
\hline$C(6)$ & $5552(4)$ & $4258(2)$ & $5559(2)$ & $67(1)$ \\
\hline $\mathrm{C}(7)$ & $6482(3)$ & $4279(1)$ & $5039(2)$ & $64(1)$ \\
\hline $\mathrm{C}(8)$ & $6615(3)$ & $3841(1)$ & $4432(2)$ & $53(1)$ \\
\hline $\mathrm{C}(9)$ & $5790(3)$ & $3349(1)$ & $4321(2)$ & $46(1)$ \\
\hline$C(10)$ & $6604(4)$ & $2662(2)$ & $3143(3)$ & $78(1)$ \\
\hline $\mathrm{C}(11)$ & $5953(3)$ & $2641(1)$ & $1998(2)$ & $66(1)$ \\
\hline$C(12)$ & $4843(2)$ & $3597(1)$ & $1760(2)$ & $39(1)$ \\
\hline$C(13)$ & $5822(3)$ & $3968(2)$ & $651(2)$ & $61(1)$ \\
\hline$C(14)$ & $6287(3)$ & $3436(2)$ & $878(3)$ & $64(1)$ \\
\hline$C(15)$ & $4156(3)$ & $4575(1)$ & $1125(2)$ & $40(1)$ \\
\hline$C(16)$ & $2888(3)$ & $4582(1)$ & 449(2) & $46(1)$ \\
\hline$C(17)$ & $2124(3)$ & $5072(1)$ & $430(2)$ & $58(1)$ \\
\hline$C(18)$ & $2585(4)$ & $5537(1)$ & $1055(3)$ & $59(1)$ \\
\hline$C(19)$ & $3868(4)$ & $5519(1)$ & $1686(2)$ & $58(1)$ \\
\hline$C(20)$ & $4688(3)$ & $5042(1)$ & $1739(2)$ & $48(1)$ \\
\hline$C(21)$ & $2358(3)$ & $4081(1)$ & $-244(2)$ & $63(1)$ \\
\hline$C(22)$ & $1720(4)$ & $6059(2)$ & $1020(3)$ & $90(1)$ \\
\hline$C(23)$ & $6092(3)$ & $5027(2)$ & $2434(3)$ & $69(1)$ \\
\hline$C(24)$ & $1823(3)$ & $2862(1)$ & $1687(2)$ & $48(1)$ \\
\hline$C(25)$ & $2439(3)$ & $4302(1)$ & $3091(2)$ & $45(1)$ \\
\hline$C(26)$ & 1911(4) & $1583(1)$ & 2031(3) & $84(1)$ \\
\hline$C(27)$ & $-696(3)$ & $2122(2)$ & 844(3) & $91(1)$ \\
\hline$C(28)$ & $388(4)$ & $2325(2)$ & $3081(3)$ & $97(1)$ \\
\hline$C(29)$ & $723(5)$ & $4208(2)$ & $4465(4)$ & $128(2)$ \\
\hline$C(30)$ & $-494(4)$ & $4022(2)$ & $2224(4)$ & $135(2)$ \\
\hline$C(31)$ & $221(4)$ & $5201(2)$ & $3004(3)$ & $83(1)$ \\
\hline
\end{tabular}


Stable 3. Anisotropic displacement parameters $\left(\AA^{2} \times 1^{3}\right)$ for 1 . The anisotropic displacement factor exponent takes the form: $-2 \pi^{2}\left[h^{2} a * 2 U^{11}+\ldots+2 h k^{*} b^{*} U^{12}\right]$

\begin{tabular}{|c|c|c|c|c|c|c|}
\hline & U11 & U22 & U33 & U23 & U13 & U12 \\
\hline $\mathrm{Y}$ & $36(1)$ & $33(1)$ & $32(1)$ & $3(1)$ & $7(1)$ & $-2(1)$ \\
\hline $\mathrm{N}(1)$ & $38(1)$ & $53(2)$ & $52(2)$ & $-16(1)$ & $9(1)$ & $4(1)$ \\
\hline $\mathrm{N}(2)$ & $35(1)$ & $51(1)$ & $42(1)$ & $-5(1)$ & $18(1)$ & $-9(1)$ \\
\hline $\operatorname{Si}(1)$ & $43(1)$ & $41(1)$ & $65(1)$ & $-9(1)$ & $15(1)$ & $-10(1)$ \\
\hline $\operatorname{Si}(2)$ & $41(1)$ & $47(1)$ & 61(1) & $2(1)$ & $11(1)$ & $7(1)$ \\
\hline$C(1)$ & $62(2)$ & $40(2)$ & $49(2)$ & $2(1)$ & $-11(2)$ & $15(2)$ \\
\hline$C(2)$ & $100(3)$ & $36(2)$ & $55(2)$ & $13(2)$ & $-22(2)$ & $-4(2)$ \\
\hline$C(3)$ & $74(2)$ & $67(2)$ & $49(2)$ & $24(2)$ & $0(2)$ & $-19(2)$ \\
\hline$C(4)$ & $57(2)$ & $61(2)$ & $31(2)$ & $10(1)$ & $-2(1)$ & $-7(2)$ \\
\hline$C(5)$ & $68(2)$ & $87(3)$ & $31(2)$ & $0(2)$ & $12(2)$ & $4(2)$ \\
\hline$C(6)$ & $81(3)$ & $68(2)$ & $42(2)$ & $-10(2)$ & $3(2)$ & $-1(2)$ \\
\hline$C(7)$ & $62(2)$ & $56(2)$ & $58(2)$ & $-6(2)$ & $-5(2)$ & $-8(2)$ \\
\hline $\mathrm{C}(8)$ & $37(2)$ & 61(2) & $50(2)$ & $1(2)$ & $-5(1)$ & $3(1)$ \\
\hline $\mathrm{C}(9)$ & $42(2)$ & $44(2)$ & $38(2)$ & $4(1)$ & $-8(1)$ & $6(1)$ \\
\hline$C(10)$ & $79(3)$ & $70(2)$ & $69(2)$ & $-10(2)$ & $-5(2)$ & $43(2)$ \\
\hline$C(11)$ & $67(2)$ & $56(2)$ & $70(2)$ & $-20(2)$ & $11(2)$ & $25(2)$ \\
\hline$C(12)$ & $32(1)$ & $41(2)$ & $40(2)$ & $-8(1)$ & $7(1)$ & $-2(1)$ \\
\hline$C(13)$ & $54(2)$ & $75(2)$ & $67(2)$ & $-13(2)$ & $38(2)$ & $-13(2)$ \\
\hline$C(14)$ & $42(2)$ & $92(3)$ & $67(2)$ & $-29(2)$ & $29(2)$ & $-4(2)$ \\
\hline$C(15)$ & $45(2)$ & $42(2)$ & $39(2)$ & $5(1)$ & $22(1)$ & $-7(1)$ \\
\hline$C(16)$ & $49(2)$ & $46(2)$ & $47(2)$ & $8(1)$ & $20(1)$ & $-7(1)$ \\
\hline$C(17)$ & $54(2)$ & $56(2)$ & $67(2)$ & $20(2)$ & $22(2)$ & $1(2)$ \\
\hline$C(18)$ & $74(2)$ & $49(2)$ & $66(2)$ & $16(2)$ & $38(2)$ & $3(2)$ \\
\hline$C(19)$ & $89(3)$ & $41(2)$ & $53(2)$ & $2(1)$ & $32(2)$ & $-8(2)$ \\
\hline$C(20)$ & $60(2)$ & $47(2)$ & $40(2)$ & $4(1)$ & $20(1)$ & $-15(2)$ \\
\hline$C(21)$ & $60(2)$ & $62(2)$ & $57(2)$ & $4(2)$ & $0(2)$ & $-13(2)$ \\
\hline$C(22)$ & $109(3)$ & $57(2)$ & $122(4)$ & $15(2)$ & $61(3)$ & $17(2)$ \\
\hline$C(23)$ & $75(2)$ & $59(2)$ & $64(2)$ & $-3(2)$ & $6(2)$ & $-21(2)$ \\
\hline$C(24)$ & $52(2)$ & $47(2)$ & $44(2)$ & $-4(1)$ & $10(1)$ & $-7(1)$ \\
\hline$C(25)$ & $51(2)$ & $45(2)$ & $43(2)$ & $-4(1)$ & $17(1)$ & $-5(1)$ \\
\hline$C(26)$ & $77(3)$ & $43(2)$ & $115(3)$ & $-17(2)$ & $4(2)$ & $-4(2)$ \\
\hline$C(27)$ & $50(2)$ & $85(3)$ & $121(3)$ & $-16(2)$ & $-2(2)$ & $-14(2)$ \\
\hline$C(28)$ & $101(3)$ & $104(3)$ & $107(3)$ & 1(3) & $64(3)$ & $-24(3)$ \\
\hline$C(29)$ & $133(4)$ & $155(5)$ & $138(4)$ & $60(4)$ & $103(4)$ & $39(4)$ \\
\hline$C(30)$ & $56(2)$ & $94(3)$ & $208(6)$ & $-55(4)$ & $-34(3)$ & $19(2)$ \\
\hline$C(31)$ & $69(2)$ & $60(2)$ & $108(3)$ & $-7(2)$ & $8(2)$ & $21(2)$ \\
\hline
\end{tabular}


Stable 4. Bond lengths $[\AA ̊]$ and angles $\left[{ }^{\circ}\right]$ for 1 .

\begin{tabular}{|c|c|c|c|}
\hline $\mathrm{Y}-\mathrm{C}(25)$ & $2.370(3)$ & $\mathrm{C}(10)-\mathrm{H}(10 \mathrm{~B})$ & 0.9900 \\
\hline $\mathrm{Y}-\mathrm{C}(24)$ & $2.370(3)$ & $\mathrm{C}(11)-\mathrm{H}(11 \mathrm{~A})$ & 0.9900 \\
\hline $\mathrm{Y}-\mathrm{C}(12)$ & $2.501(3)$ & $\mathrm{C}(11)-\mathrm{H}(11 \mathrm{~B})$ & 0.9900 \\
\hline $\mathrm{Y}-\mathrm{C}(3)$ & $2.640(3)$ & $C(13)-C(14)$ & $1.334(4)$ \\
\hline $\mathrm{Y}-\mathrm{C}(2)$ & $2.672(3)$ & $\mathrm{C}(13)-\mathrm{H}(13)$ & 0.9500 \\
\hline $\mathrm{Y}-\mathrm{C}(4)$ & $2.686(3)$ & $\mathrm{C}(14)-\mathrm{H}(14)$ & 0.9500 \\
\hline $\mathrm{Y}-\mathrm{C}(9)$ & $2.700(3)$ & $C(15)-C(16)$ & $1.390(4)$ \\
\hline $\mathrm{Y}-\mathrm{C}(1)$ & $2.709(3)$ & $C(15)-C(20)$ & $1.394(4)$ \\
\hline $\mathrm{N}(1)-\mathrm{C}(12)$ & $1.360(3)$ & $\mathrm{C}(16)-\mathrm{C}(17)$ & $1.394(4)$ \\
\hline $\mathrm{N}(1)-\mathrm{C}(14)$ & $1.373(4)$ & $C(16)-C(21)$ & $1.509(4)$ \\
\hline $\mathrm{N}(1)-\mathrm{C}(11)$ & $1.460(4)$ & $C(17)-C(18)$ & $1.382(4)$ \\
\hline $\mathrm{N}(2)-\mathrm{C}(12)$ & $1.355(3)$ & $\mathrm{C}(17)-\mathrm{H}(17)$ & 0.9500 \\
\hline $\mathrm{N}(2)-\mathrm{C}(13)$ & $1.380(3)$ & $C(18)-C(19)$ & $1.380(5)$ \\
\hline $\mathrm{N}(2)-\mathrm{C}(15)$ & $1.442(3)$ & $C(18)-C(22)$ & $1.511(4)$ \\
\hline $\operatorname{Si}(1)-C(24)$ & $1.824(3)$ & $C(19)-C(20)$ & $1.395(4)$ \\
\hline $\mathrm{Si}(1)-\mathrm{C}(26)$ & $1.871(3)$ & $\mathrm{C}(19)-\mathrm{H}(19)$ & 0.9500 \\
\hline $\operatorname{Si}(1)-C(27)$ & $1.872(4)$ & $C(20)-C(23)$ & $1.512(4)$ \\
\hline $\operatorname{Si}(1)-C(28)$ & $1.879(4)$ & $\mathrm{C}(21)-\mathrm{H}(21 \mathrm{~A})$ & 0.9800 \\
\hline $\operatorname{Si}(2)-C(25)$ & $1.816(3)$ & $\mathrm{C}(21)-\mathrm{H}(21 \mathrm{~B})$ & 0.9800 \\
\hline $\mathrm{Si}(2)-\mathrm{C}(29)$ & $1.862(4)$ & $\mathrm{C}(21)-\mathrm{H}(21 \mathrm{C})$ & 0.9800 \\
\hline $\operatorname{Si}(2)-C(30)$ & $1.873(4)$ & $\mathrm{C}(22)-\mathrm{H}(22 \mathrm{~A})$ & 0.9800 \\
\hline $\operatorname{Si}(2)-C(31)$ & $1.876(3)$ & $\mathrm{C}(22)-\mathrm{H}(22 \mathrm{~B})$ & 0.9800 \\
\hline $\mathrm{C}(1)-\mathrm{C}(2)$ & $1.393(5)$ & $\mathrm{C}(22)-\mathrm{H}(22 \mathrm{C})$ & 0.9800 \\
\hline $\mathrm{C}(1)-\mathrm{C}(9)$ & $1.424(4)$ & $\mathrm{C}(23)-\mathrm{H}(23 \mathrm{~A})$ & 0.9800 \\
\hline$C(1)-C(10)$ & $1.500(5)$ & $\mathrm{C}(23)-\mathrm{H}(23 \mathrm{~B})$ & 0.9800 \\
\hline $\mathrm{C}(2)-\mathrm{C}(3)$ & $1.413(5)$ & $\mathrm{C}(23)-\mathrm{H}(23 \mathrm{C})$ & 0.9800 \\
\hline $\mathrm{C}(2)-\mathrm{H}(2)$ & 0.9500 & $\mathrm{C}(24)-\mathrm{H}(24 \mathrm{~A})$ & 0.9900 \\
\hline$C(3)-C(4)$ & $1.424(4)$ & $\mathrm{C}(24)-\mathrm{H}(24 \mathrm{~B})$ & 0.9900 \\
\hline $\mathrm{C}(3)-\mathrm{H}(3)$ & 0.9500 & $\mathrm{C}(25)-\mathrm{H}(25 \mathrm{~A})$ & 0.9900 \\
\hline $\mathrm{C}(4)-\mathrm{C}(9)$ & $1.425(4)$ & $\mathrm{C}(25)-\mathrm{H}(25 \mathrm{~B})$ & 0.9900 \\
\hline$C(4)-C(5)$ & $1.433(4)$ & $\mathrm{C}(26)-\mathrm{H}(26 \mathrm{~A})$ & 0.9800 \\
\hline$C(5)-C(6)$ & $1.357(5)$ & $\mathrm{C}(26)-\mathrm{H}(26 \mathrm{~B})$ & 0.9800 \\
\hline $\mathrm{C}(5)-\mathrm{H}(5)$ & 0.9500 & $\mathrm{C}(26)-\mathrm{H}(26 \mathrm{C})$ & 0.9800 \\
\hline$C(6)-C(7)$ & $1.384(5)$ & $\mathrm{C}(27)-\mathrm{H}(27 \mathrm{~A})$ & 0.9800 \\
\hline $\mathrm{C}(6)-\mathrm{H}(6)$ & 0.9500 & $\mathrm{C}(27)-\mathrm{H}(27 \mathrm{~B})$ & 0.9800 \\
\hline $\mathrm{C}(7)-\mathrm{C}(8)$ & $1.357(4)$ & $\mathrm{C}(27)-\mathrm{H}(27 \mathrm{C})$ & 0.9800 \\
\hline $\mathrm{C}(7)-\mathrm{H}(7)$ & 0.9500 & $\mathrm{C}(28)-\mathrm{H}(28 \mathrm{~A})$ & 0.9800 \\
\hline $\mathrm{C}(8)-\mathrm{C}(9)$ & $1.418(4)$ & $\mathrm{C}(28)-\mathrm{H}(28 \mathrm{~B})$ & 0.9800 \\
\hline $\mathrm{C}(8)-\mathrm{H}(8)$ & 0.9500 & $\mathrm{C}(28)-\mathrm{H}(28 \mathrm{C})$ & 0.9800 \\
\hline$C(10)-C(11)$ & $1.538(4)$ & $\mathrm{C}(29)-\mathrm{H}(29 \mathrm{~A})$ & 0.9800 \\
\hline $\mathrm{C}(10)-\mathrm{H}(10 \mathrm{~A})$ & 0.9900 & $\mathrm{C}(29)-\mathrm{H}(29 \mathrm{~B})$ & 0.9800 \\
\hline
\end{tabular}




\begin{tabular}{|c|c|c|c|}
\hline C(29)-H(29C) & 0.9800 & $\mathrm{C}(30)-\mathrm{H}(30 \mathrm{C})$ & 0.9800 \\
\hline $\mathrm{C}(30)-\mathrm{H}(30 \mathrm{~A})$ & 0.9800 & $\mathrm{C}(31)-\mathrm{H}(31 \mathrm{~A})$ & 0.9800 \\
\hline $\mathrm{C}(30)-\mathrm{H}(30 \mathrm{~B})$ & 0.9800 & $\mathrm{C}(31)-\mathrm{H}(31 \mathrm{~B})$ & 0.9800 \\
\hline \multirow[t]{2}{*}{$\mathrm{C}(31)-\mathrm{H}(31 \mathrm{C})$} & 0.9800 & & \\
\hline & & $C(27)-\operatorname{Si}(1)-C(28)$ & $107.15(19)$ \\
\hline$C(25)-Y-C(24)$ & $107.88(10)$ & $C(25)-\operatorname{Si}(2)-C(29)$ & $109.66(18)$ \\
\hline$C(25)-Y-C(12)$ & $107.11(9)$ & $C(25)-\operatorname{Si}(2)-C(30)$ & $111.19(18)$ \\
\hline $\mathrm{C}(24)-\mathrm{Y}-\mathrm{C}(12)$ & 95.06(9) & $C(29)-\operatorname{Si}(2)-C(30)$ & $108.5(3)$ \\
\hline C(25)-Y-C(3) & $111.58(11)$ & $C(25)-\operatorname{Si}(2)-C(31)$ & $115.13(16)$ \\
\hline$C(24)-Y-C(3)$ & $106.62(10)$ & $C(29)-\operatorname{Si}(2)-C(31)$ & $107.6(2)$ \\
\hline$C(12)-Y-C(3)$ & $126.23(11)$ & $C(30)-\operatorname{Si}(2)-C(31)$ & $104.49(19)$ \\
\hline$C(25)-Y-C(2)$ & $141.80(11)$ & $\mathrm{C}(2)-\mathrm{C}(1)-\mathrm{C}(9)$ & $106.3(3)$ \\
\hline$C(24)-Y-C(2)$ & $95.41(10)$ & $C(2)-C(1)-C(10)$ & $127.1(3)$ \\
\hline$C(12)-Y-C(2)$ & $100.29(12)$ & $C(9)-C(1)-C(10)$ & $126.5(3)$ \\
\hline C(3)-Y-C(2) & $30.85(11)$ & $C(2)-C(1)-Y$ & $73.54(18)$ \\
\hline$C(25)-Y-C(4)$ & $93.57(10)$ & $C(9)-C(1)-Y$ & $74.39(15)$ \\
\hline$C(24)-Y-C(4)$ & $137.50(9)$ & $C(10)-C(1)-Y$ & $120.62(19)$ \\
\hline$C(12)-Y-C(4)$ & $113.47(9)$ & $\mathrm{C}(1)-\mathrm{C}(2)-\mathrm{C}(3)$ & $110.4(3)$ \\
\hline C(3)-Y-C(4) & $31.00(9)$ & $\mathrm{C}(1)-\mathrm{C}(2)-\mathrm{Y}$ & $76.47(17)$ \\
\hline C(2)-Y-C(4) & $50.52(10)$ & $C(3)-C(2)-Y$ & $73.31(18)$ \\
\hline $\mathrm{C}(25)-\mathrm{Y}-\mathrm{C}(9)$ & 107.61(9) & $\mathrm{C}(1)-\mathrm{C}(2)-\mathrm{H}(2)$ & 124.8 \\
\hline$C(24)-Y-C(9)$ & $143.34(9)$ & $\mathrm{C}(3)-\mathrm{C}(2)-\mathrm{H}(2)$ & 124.8 \\
\hline$C(12)-Y-C(9)$ & 83.11(9) & $\mathrm{Y}-\mathrm{C}(2)-\mathrm{H}(2)$ & 117.2 \\
\hline C(3)-Y-C(9) & $50.63(10)$ & $C(2)-C(3)-C(4)$ & 107.4(3) \\
\hline $\mathrm{C}(2)-\mathrm{Y}-\mathrm{C}(9)$ & $49.60(9)$ & $\mathrm{C}(2)-\mathrm{C}(3)-\mathrm{Y}$ & $75.84(17)$ \\
\hline C(4)-Y-C(9) & $30.69(9)$ & $C(4)-C(3)-Y$ & $76.31(16)$ \\
\hline$C(25)-Y-C(1)$ & 138.12(9) & $\mathrm{C}(2)-\mathrm{C}(3)-\mathrm{H}(3)$ & 126.3 \\
\hline$C(24)-Y-C(1)$ & $113.60(10)$ & $\mathrm{C}(4)-\mathrm{C}(3)-\mathrm{H}(3)$ & 126.3 \\
\hline $\mathrm{C}(12)-\mathrm{Y}-\mathrm{C}(1)$ & $75.25(10)$ & $\mathrm{Y}-\mathrm{C}(3)-\mathrm{H}(3)$ & 114.0 \\
\hline C(3)-Y-C(1) & $51.00(11)$ & $\mathrm{C}(3)-\mathrm{C}(4)-\mathrm{C}(9)$ & $106.6(3)$ \\
\hline$C(2)-Y-C(1)$ & $29.99(10)$ & $C(3)-C(4)-C(5)$ & $133.6(3)$ \\
\hline $\mathrm{C}(4)-\mathrm{Y}-\mathrm{C}(1)$ & $51.05(10)$ & $C(9)-C(4)-C(5)$ & 119.8(3) \\
\hline C(9)-Y-C(1) & $30.53(8)$ & $C(3)-C(4)-Y$ & $72.69(16)$ \\
\hline $\mathrm{C}(12)-\mathrm{N}(1)-\mathrm{C}(14)$ & $111.2(3)$ & $\mathrm{C}(9)-\mathrm{C}(4)-\mathrm{Y}$ & $75.19(15)$ \\
\hline $\mathrm{C}(12)-\mathrm{N}(1)-\mathrm{C}(11)$ & $124.0(3)$ & $C(5)-C(4)-Y$ & $116.78(19)$ \\
\hline $\mathrm{C}(14)-\mathrm{N}(1)-\mathrm{C}(11)$ & $124.8(3)$ & $C(6)-C(5)-C(4)$ & $118.2(3)$ \\
\hline $\mathrm{C}(12)-\mathrm{N}(2)-\mathrm{C}(13)$ & $112.0(3)$ & $\mathrm{C}(6)-\mathrm{C}(5)-\mathrm{H}(5)$ & 120.9 \\
\hline $\mathrm{C}(12)-\mathrm{N}(2)-\mathrm{C}(15)$ & $123.9(2)$ & $\mathrm{C}(4)-\mathrm{C}(5)-\mathrm{H}(5)$ & 120.9 \\
\hline $\mathrm{C}(13)-\mathrm{N}(2)-\mathrm{C}(15)$ & $124.0(2)$ & $C(5)-C(6)-C(7)$ & $122.2(3)$ \\
\hline $\mathrm{C}(24)-\mathrm{Si}(1)-\mathrm{C}(26)$ & $109.13(16)$ & $\mathrm{C}(5)-\mathrm{C}(6)-\mathrm{H}(6)$ & 118.9 \\
\hline $\mathrm{C}(24)-\mathrm{Si}(1)-\mathrm{C}(27)$ & $113.59(17)$ & $\mathrm{C}(7)-\mathrm{C}(6)-\mathrm{H}(6)$ & 118.9 \\
\hline $\mathrm{C}(26)-\mathrm{Si}(1)-\mathrm{C}(27)$ & $108.16(17)$ & $\mathrm{C}(8)-\mathrm{C}(7)-\mathrm{C}(6)$ & $121.4(3)$ \\
\hline $\mathrm{C}(24)-\mathrm{Si}(1)-\mathrm{C}(28)$ & $111.48(16)$ & $\mathrm{C}(8)-\mathrm{C}(7)-\mathrm{H}(7)$ & 119.3 \\
\hline $\mathrm{C}(26)-\mathrm{Si}(1)-\mathrm{C}(28)$ & $107.1(2)$ & $\mathrm{C}(6)-\mathrm{C}(7)-\mathrm{H}(7)$ & 119.3 \\
\hline
\end{tabular}




\begin{tabular}{|c|c|c|c|}
\hline $\mathrm{C}(7)-\mathrm{C}(8)-\mathrm{C}(9)$ & $119.9(3)$ & $\mathrm{C}(20)-\mathrm{C}(19)-\mathrm{H}(19)$ & 118.8 \\
\hline $\mathrm{C}(7)-\mathrm{C}(8)-\mathrm{H}(8)$ & 120.0 & $C(15)-C(20)-C(19)$ & $117.2(3)$ \\
\hline $\mathrm{C}(9)-\mathrm{C}(8)-\mathrm{H}(8)$ & 120.0 & $C(15)-C(20)-C(23)$ & $121.0(3)$ \\
\hline $\mathrm{C}(8)-\mathrm{C}(9)-\mathrm{C}(1)$ & $132.2(3)$ & $C(19)-C(20)-C(23)$ & $121.8(3)$ \\
\hline $\mathrm{C}(8)-\mathrm{C}(9)-\mathrm{C}(4)$ & $118.4(3)$ & $\mathrm{C}(16)-\mathrm{C}(21)-\mathrm{H}(21 \mathrm{~A})$ & 109.5 \\
\hline$C(1)-C(9)-C(4)$ & $109.4(3)$ & $\mathrm{C}(16)-\mathrm{C}(21)-\mathrm{H}(21 \mathrm{~B})$ & 109.5 \\
\hline$C(8)-C(9)-Y$ & $116.06(17)$ & $\mathrm{H}(21 \mathrm{~A})-\mathrm{C}(21)-\mathrm{H}(21 \mathrm{~B})$ & 109.5 \\
\hline$C(1)-C(9)-Y$ & $75.08(15)$ & $\mathrm{C}(16)-\mathrm{C}(21)-\mathrm{H}(21 \mathrm{C})$ & 109.5 \\
\hline$C(4)-C(9)-Y$ & $74.13(15)$ & $\mathrm{H}(21 \mathrm{~A})-\mathrm{C}(21)-\mathrm{H}(21 \mathrm{C})$ & 109.5 \\
\hline $\mathrm{C}(1)-\mathrm{C}(10)-\mathrm{C}(11)$ & $115.5(3)$ & $\mathrm{H}(21 \mathrm{~B})-\mathrm{C}(21)-\mathrm{H}(21 \mathrm{C})$ & 109.5 \\
\hline $\mathrm{C}(1)-\mathrm{C}(10)-\mathrm{H}(10 \mathrm{~A})$ & 108.4 & $\mathrm{C}(18)-\mathrm{C}(22)-\mathrm{H}(22 \mathrm{~A})$ & 109.5 \\
\hline $\mathrm{C}(11)-\mathrm{C}(10)-\mathrm{H}(10 \mathrm{~A})$ & 108.4 & $\mathrm{C}(18)-\mathrm{C}(22)-\mathrm{H}(22 \mathrm{~B})$ & 109.5 \\
\hline $\mathrm{C}(1)-\mathrm{C}(10)-\mathrm{H}(10 \mathrm{~B})$ & 108.4 & $\mathrm{H}(22 \mathrm{~A})-\mathrm{C}(22)-\mathrm{H}(22 \mathrm{~B})$ & 109.5 \\
\hline $\mathrm{C}(11)-\mathrm{C}(10)-\mathrm{H}(10 \mathrm{~B})$ & 108.4 & $\mathrm{C}(18)-\mathrm{C}(22)-\mathrm{H}(22 \mathrm{C})$ & 109.5 \\
\hline $\mathrm{H}(10 \mathrm{~A})-\mathrm{C}(10)-\mathrm{H}(10 \mathrm{~B})$ & 107.5 & $\mathrm{H}(22 \mathrm{~A})-\mathrm{C}(22)-\mathrm{H}(22 \mathrm{C})$ & 109.5 \\
\hline $\mathrm{N}(1)-\mathrm{C}(11)-\mathrm{C}(10)$ & $112.2(3)$ & $\mathrm{H}(22 \mathrm{~B})-\mathrm{C}(22)-\mathrm{H}(22 \mathrm{C})$ & 109.5 \\
\hline $\mathrm{N}(1)-\mathrm{C}(11)-\mathrm{H}(11 \mathrm{~A})$ & 109.2 & $\mathrm{C}(20)-\mathrm{C}(23)-\mathrm{H}(23 \mathrm{~A})$ & 109.5 \\
\hline $\mathrm{C}(10)-\mathrm{C}(11)-\mathrm{H}(11 \mathrm{~A})$ & 109.2 & $\mathrm{C}(20)-\mathrm{C}(23)-\mathrm{H}(23 \mathrm{~B})$ & 109.5 \\
\hline $\mathrm{N}(1)-\mathrm{C}(11)-\mathrm{H}(11 \mathrm{~B})$ & 109.2 & $\mathrm{H}(23 \mathrm{~A})-\mathrm{C}(23)-\mathrm{H}(23 \mathrm{~B})$ & 109.5 \\
\hline C(10)-C(11)-H(11B) & 109.2 & $\mathrm{C}(20)-\mathrm{C}(23)-\mathrm{H}(23 \mathrm{C})$ & 109.5 \\
\hline $\mathrm{H}(11 \mathrm{~A})-\mathrm{C}(11)-\mathrm{H}(11 \mathrm{~B})$ & 107.9 & $\mathrm{H}(23 \mathrm{~A})-\mathrm{C}(23)-\mathrm{H}(23 \mathrm{C})$ & 109.5 \\
\hline $\mathrm{N}(2)-\mathrm{C}(12)-\mathrm{N}(1)$ & $103.2(2)$ & $\mathrm{H}(23 \mathrm{~B})-\mathrm{C}(23)-\mathrm{H}(23 \mathrm{C})$ & 109.5 \\
\hline $\mathrm{N}(2)-\mathrm{C}(12)-\mathrm{Y}$ & $131.39(18)$ & $\mathrm{Si}(1)-\mathrm{C}(24)-\mathrm{Y}$ & $129.00(14)$ \\
\hline$N(1)-C(12)-Y$ & $125.4(2)$ & $\mathrm{Si}(1)-\mathrm{C}(24)-\mathrm{H}(24 \mathrm{~A})$ & 105.0 \\
\hline $\mathrm{C}(14)-\mathrm{C}(13)-\mathrm{N}(2)$ & $106.0(3)$ & $\mathrm{Y}-\mathrm{C}(24)-\mathrm{H}(24 \mathrm{~A})$ & 105.0 \\
\hline $\mathrm{C}(14)-\mathrm{C}(13)-\mathrm{H}(13)$ & 127.0 & $\mathrm{Si}(1)-\mathrm{C}(24)-\mathrm{H}(24 \mathrm{~B})$ & 105.0 \\
\hline $\mathrm{N}(2)-\mathrm{C}(13)-\mathrm{H}(13)$ & 127.0 & $\mathrm{Y}-\mathrm{C}(24)-\mathrm{H}(24 \mathrm{~B})$ & 105.0 \\
\hline $\mathrm{C}(13)-\mathrm{C}(14)-\mathrm{N}(1)$ & $107.5(3)$ & $\mathrm{H}(24 \mathrm{~A})-\mathrm{C}(24)-\mathrm{H}(24 \mathrm{~B})$ & 105.9 \\
\hline $\mathrm{C}(13)-\mathrm{C}(14)-\mathrm{H}(14)$ & 126.2 & $\mathrm{Si}(2)-\mathrm{C}(25)-\mathrm{Y}$ & $128.71(14)$ \\
\hline $\mathrm{N}(1)-\mathrm{C}(14)-\mathrm{H}(14)$ & 126.2 & $\mathrm{Si}(2)-\mathrm{C}(25)-\mathrm{H}(25 \mathrm{~A})$ & 105.1 \\
\hline$C(16)-C(15)-C(20)$ & $122.6(3)$ & $\mathrm{Y}-\mathrm{C}(25)-\mathrm{H}(25 \mathrm{~A})$ & 105.1 \\
\hline $\mathrm{C}(16)-\mathrm{C}(15)-\mathrm{N}(2)$ & $118.3(2)$ & $\mathrm{Si}(2)-\mathrm{C}(25)-\mathrm{H}(25 \mathrm{~B})$ & 105.1 \\
\hline $\mathrm{C}(20)-\mathrm{C}(15)-\mathrm{N}(2)$ & $119.1(2)$ & $\mathrm{Y}-\mathrm{C}(25)-\mathrm{H}(25 \mathrm{~B})$ & 105.1 \\
\hline$C(15)-C(16)-C(17)$ & $117.3(3)$ & $\mathrm{H}(25 \mathrm{~A})-\mathrm{C}(25)-\mathrm{H}(25 \mathrm{~B})$ & 105.9 \\
\hline$C(15)-C(16)-C(21)$ & $121.6(3)$ & $\mathrm{Si}(1)-\mathrm{C}(26)-\mathrm{H}(26 \mathrm{~A})$ & 109.5 \\
\hline $\mathrm{C}(17)-\mathrm{C}(16)-\mathrm{C}(21)$ & $121.1(3)$ & $\mathrm{Si}(1)-\mathrm{C}(26)-\mathrm{H}(26 \mathrm{~B})$ & 109.5 \\
\hline$C(18)-C(17)-C(16)$ & $122.3(3)$ & $\mathrm{H}(26 \mathrm{~A})-\mathrm{C}(26)-\mathrm{H}(26 \mathrm{~B})$ & 109.5 \\
\hline $\mathrm{C}(18)-\mathrm{C}(17)-\mathrm{H}(17)$ & 118.9 & $\mathrm{Si}(1)-\mathrm{C}(26)-\mathrm{H}(26 \mathrm{C})$ & 109.5 \\
\hline $\mathrm{C}(16)-\mathrm{C}(17)-\mathrm{H}(17)$ & 118.9 & $\mathrm{H}(26 \mathrm{~A})-\mathrm{C}(26)-\mathrm{H}(26 \mathrm{C})$ & 109.5 \\
\hline$C(19)-C(18)-C(17)$ & $118.2(3)$ & $\mathrm{H}(26 \mathrm{~B})-\mathrm{C}(26)-\mathrm{H}(26 \mathrm{C})$ & 109.5 \\
\hline $\mathrm{C}(19)-\mathrm{C}(18)-\mathrm{C}(22)$ & $121.0(3)$ & $\mathrm{Si}(1)-\mathrm{C}(27)-\mathrm{H}(27 \mathrm{~A})$ & 109.5 \\
\hline $\mathrm{C}(17)-\mathrm{C}(18)-\mathrm{C}(22)$ & $120.7(4)$ & $\mathrm{Si}(1)-\mathrm{C}(27)-\mathrm{H}(27 \mathrm{~B})$ & 109.5 \\
\hline$C(18)-C(19)-C(20)$ & $122.3(3)$ & $\mathrm{H}(27 \mathrm{~A})-\mathrm{C}(27)-\mathrm{H}(27 \mathrm{~B})$ & 109.5 \\
\hline C(18)-C(19)-H(19) & 118.8 & $\mathrm{Si}(1)-\mathrm{C}(27)-\mathrm{H}(27 \mathrm{C})$ & 109.5 \\
\hline
\end{tabular}


$\mathrm{Si}(1)-\mathrm{C}(28)-\mathrm{H}(28 \mathrm{~B})$

109.5

$\mathrm{H}(28 \mathrm{~A})-\mathrm{C}(28)-\mathrm{H}(28 \mathrm{~B})$

109.5

$\mathrm{Si}(1)-\mathrm{C}(28)-\mathrm{H}(28 \mathrm{C})$

109.5

$\mathrm{H}(27 \mathrm{~A})-\mathrm{C}(27)-\mathrm{H}(27 \mathrm{C})$

109.5

$\mathrm{H}(28 \mathrm{~A})-\mathrm{C}(28)-\mathrm{H}(28 \mathrm{C})$

109.5

$\mathrm{H}(27 \mathrm{~B})-\mathrm{C}(27)-\mathrm{H}(27 \mathrm{C})$

109.5

$\mathrm{H}(28 \mathrm{~B})-\mathrm{C}(28)-\mathrm{H}(28 \mathrm{C})$

109.5

$\mathrm{Si}(1)-\mathrm{C}(28)-\mathrm{H}(28 \mathrm{~A})$

109.5 
Stable 5. Crystal data and structure refinement for 2

\begin{tabular}{|c|c|c|}
\hline Identification code & \multicolumn{2}{|l|}{$\mathrm{q} 473 \mathrm{~m}$} \\
\hline Empirical formula & \multicolumn{2}{|c|}{ C31 H45 Lu N2 Si2 } \\
\hline Formula weight & \multicolumn{2}{|l|}{676.84} \\
\hline Temperature & \multicolumn{2}{|l|}{$187(2) \mathrm{K}$} \\
\hline Wavelength & \multicolumn{2}{|l|}{$0.71073 \AA$} \\
\hline Crystal system & \multicolumn{2}{|l|}{ Monoclinic } \\
\hline Space group & \multicolumn{2}{|l|}{$\mathrm{P} 2{ }_{1} / \mathrm{c}$} \\
\hline \multirow[t]{3}{*}{ Unit cell dimensions } & $\mathrm{a}=10.5025(6) \AA$ & $\alpha=90^{\circ}$ \\
\hline & $\mathrm{b}=23.2946(13) \AA$ & $\beta=106.8380(10)^{\circ}$. \\
\hline & $\mathrm{c}=13.8088(7) \AA$ & $\gamma=90^{\circ}$. \\
\hline Volume & \multicolumn{2}{|l|}{$3233.5(3) \AA^{3}$} \\
\hline $\mathrm{Z}$ & \multicolumn{2}{|l|}{4} \\
\hline Density (calculated) & \multicolumn{2}{|l|}{$1.390 \mathrm{Mg} / \mathrm{m}^{3}$} \\
\hline Absorption coefficient & \multicolumn{2}{|l|}{$3.148 \mathrm{~mm}^{-1}$} \\
\hline $\mathrm{F}(000)$ & \multicolumn{2}{|l|}{1376} \\
\hline Crystal size & \multicolumn{2}{|c|}{$0.17 \times 0.16 \times 0.10 \mathrm{~mm}^{3}$} \\
\hline Theta range for data collection & \multicolumn{2}{|c|}{1.75 to $26.05^{\circ}$. } \\
\hline Index ranges & \multicolumn{2}{|c|}{$-12<=\mathrm{h}<=12,-28<=\mathrm{k}<=28,-17<=1<=8$} \\
\hline Reflections collected & \multicolumn{2}{|l|}{17999} \\
\hline Independent reflections & \multicolumn{2}{|c|}{$6374[\mathrm{R}(\mathrm{int})=0.0505]$} \\
\hline Completeness to theta $=26.05^{\circ}$ & \multicolumn{2}{|l|}{$99.8 \%$} \\
\hline Absorption correction & \multicolumn{2}{|c|}{ Semi-empirical from equivalents } \\
\hline Max. and min. transmission & \multicolumn{2}{|c|}{0.7416 and 0.6247} \\
\hline Refinement method & \multicolumn{2}{|c|}{ Full-matrix least-squares on $\mathrm{F}^{2}$} \\
\hline Data / restraints / parameters & \multicolumn{2}{|c|}{$6374 / 0 / 334$} \\
\hline Goodness-of-fit on $\mathrm{F}^{2}$ & \multicolumn{2}{|l|}{0.956} \\
\hline Final $\mathrm{R}$ indices [I>2sigma $(\mathrm{I})]$ & \multicolumn{2}{|c|}{$\mathrm{R} 1=0.0260, \mathrm{wR} 2=0.0598$} \\
\hline $\mathrm{R}$ indices (all data) & \multicolumn{2}{|c|}{$\mathrm{R} 1=0.0336, \mathrm{wR} 2=0.0618$} \\
\hline Largest diff. peak and hole & \multicolumn{2}{|c|}{0.946 and $-0.610 \mathrm{e}^{-3}$} \\
\hline
\end{tabular}

Stable 6. Atomic coordinates ( $\left.x 1^{4}\right)$ and equivalent isotropic displacement parameters $\left(\AA^{2} x\right.$ $10^{3}$ ) for complex 2. $U(\mathrm{eq})$ is defined as one third of the trace of the orthogonalized $U^{i j}$ tensor.

\begin{tabular}{lrrrr}
\hline & $\mathrm{x}$ & $\mathrm{y}$ & $\mathrm{z}$ & $\mathrm{U}(\mathrm{eq})$ \\
\hline $\mathrm{Lu}$ & $1596(1)$ & $3412(1)$ & $7110(1)$ & $32(1)$ \\
$\mathrm{Si}(2)$ & $4227(1)$ & $4422(1)$ & $6809(1)$ & $44(1)$ \\
$\mathrm{Si}(1)$ & $4144(1)$ & $2258(1)$ & $8087(1)$ & $47(1)$ \\
$\mathrm{N}(2)$ & $91(2)$ & $4047(1)$ & $8798(2)$ & $38(1)$
\end{tabular}




\begin{tabular}{|c|c|c|c|c|}
\hline $\mathrm{N}(1)$ & $-630(3)$ & $3193(1)$ & $8437(2)$ & $46(1)$ \\
\hline$C(15)$ & $859(3)$ & $4566(1)$ & $8872(2)$ & $38(1)$ \\
\hline$C(14)$ & $-1241(4)$ & $3404(2)$ & $9120(3)$ & $62(1)$ \\
\hline$C(12)$ & $206(3)$ & $3588(2)$ & $8225(2)$ & $37(1)$ \\
\hline$C(13)$ & $-793(4)$ & $3940(2)$ & 9349(3) & $59(1)$ \\
\hline $\mathrm{C}(9)$ & $-744(3)$ & $3354(1)$ & $5677(2)$ & $42(1)$ \\
\hline$C(4)$ & $264(4)$ & $3368(1)$ & $5160(2)$ & $46(1)$ \\
\hline$C(8)$ & $-1620(3)$ & $3829(2)$ & $5575(3)$ & $50(1)$ \\
\hline$C(7)$ & $-1513(4)$ & $4273(2)$ & 4975(3) & $58(1)$ \\
\hline $\mathrm{C}(1)$ & $-621(4)$ & $2831(2)$ & $6235(3)$ & $56(1)$ \\
\hline$C(5)$ & $329(4)$ & $3847(2)$ & $4541(3)$ & $57(1)$ \\
\hline$C(6)$ & $-550(4)$ & $4281(2)$ & $4454(3)$ & $59(1)$ \\
\hline$C(11)$ & $-848(4)$ & $2619(2)$ & 7999(3) & $65(1)$ \\
\hline $\mathrm{C}(2)$ & $448(4)$ & $2533(2)$ & $6042(3)$ & $68(1)$ \\
\hline$C(3)$ & $1009(4)$ & $2857(2)$ & $5419(3)$ & $61(1)$ \\
\hline$C(10)$ & $-1495(4)$ & $2628(2)$ & 6853(3) & $83(2)$ \\
\hline$C(25)$ & 2587(3) & $4282(1)$ & $6947(2)$ & $40(1)$ \\
\hline$C(24)$ & $3179(3)$ & 2879(1) & $8285(2)$ & $42(1)$ \\
\hline$C(26)$ & 3099(5) & 1594(2) & $7980(4)$ & $75(1)$ \\
\hline$C(31)$ & $4782(4)$ & $5188(2)$ & 7021(3) & $68(1)$ \\
\hline$C(27)$ & 5708(4) & $2147(2)$ & $9139(4)$ & $86(2)$ \\
\hline$C(30)$ & $5550(4)$ & $3997(2)$ & $7740(4)$ & $107(2)$ \\
\hline$C(29)$ & $4273(5)$ & $4216(2)$ & $5508(4)$ & $98(2)$ \\
\hline$C(28)$ & $4643(5)$ & $2318(2)$ & $6893(4)$ & $90(2)$ \\
\hline$C(16)$ & 2129(3) & $4573(2)$ & $9564(3)$ & $44(1)$ \\
\hline$C(20)$ & $324(3)$ & $5031(2)$ & $8258(2)$ & $44(1)$ \\
\hline$C(19)$ & $1137(4)$ & $5510(2)$ & $8323(3)$ & $52(1)$ \\
\hline$C(17)$ & 2883(4) & $5066(2)$ & $9585(3)$ & $52(1)$ \\
\hline$C(18)$ & $2425(4)$ & $5531(2)$ & $8971(3)$ & $54(1)$ \\
\hline$C(21)$ & $2658(4)$ & $4075(2)$ & $10246(3)$ & $59(1)$ \\
\hline$C(23)$ & $-1087(4)$ & $5010(2)$ & $7562(3)$ & $66(1)$ \\
\hline $\mathrm{C}(22)$ & $3271(5)$ & $6059(2)$ & $8998(4)$ & $81(1)$ \\
\hline
\end{tabular}

Stable 7. Anisotropic displacement parameters $\left(\AA^{2} \times 10^{3}\right)$ for 2 . The anisotropic displacement factor exponent takes the form: $-2 \pi^{2}\left[h^{2} a * 2 U^{11}+\ldots+2 h k^{*} b^{*} U^{12}\right]$

\begin{tabular}{lcccrrr}
\hline & U11 & U22 & U33 & U23 & U13 & U12 \\
\hline $\mathrm{Lu}$ & $35(1)$ & $30(1)$ & $28(1)$ & $-3(1)$ & $6(1)$ & $2(1)$ \\
$\mathrm{Si}(2)$ & $39(1)$ & $43(1)$ & $50(1)$ & $0(1)$ & $10(1)$ & $-6(1)$ \\
$\mathrm{Si}(1)$ & $42(1)$ & $41(1)$ & $57(1)$ & $9(1)$ & $16(1)$ & $11(1)$ \\
$\mathrm{N}(2)$ & $35(1)$ & $47(2)$ & $37(2)$ & $5(1)$ & $17(1)$ & $6(1)$
\end{tabular}




\begin{tabular}{|c|c|c|c|c|c|c|}
\hline $\mathrm{N}(1)$ & $37(2)$ & $50(2)$ & $49(2)$ & $18(2)$ & $7(1)$ & $-7(1)$ \\
\hline$C(15)$ & $45(2)$ & $40(2)$ & $35(2)$ & $-5(2)$ & $22(2)$ & $6(2)$ \\
\hline$C(14)$ & $40(2)$ & $87(4)$ & $66(3)$ & $27(2)$ & $27(2)$ & $4(2)$ \\
\hline$C(12)$ & $31(2)$ & $41(2)$ & $37(2)$ & $11(2)$ & $6(1)$ & $0(1)$ \\
\hline$C(13)$ & $51(2)$ & $84(3)$ & $56(3)$ & $18(2)$ & $35(2)$ & $17(2)$ \\
\hline$C(9)$ & $41(2)$ & $40(2)$ & $31(2)$ & $-2(2)$ & $-10(2)$ & $-5(2)$ \\
\hline $\mathrm{C}(4)$ & $55(2)$ & $45(2)$ & $29(2)$ & $-11(2)$ & $-2(2)$ & $8(2)$ \\
\hline C(8) & $35(2)$ & $56(3)$ & $49(2)$ & $-2(2)$ & $-1(2)$ & $0(2)$ \\
\hline $\mathrm{C}(7)$ & $59(2)$ & $52(3)$ & $48(2)$ & $3(2)$ & $-6(2)$ & $12(2)$ \\
\hline $\mathrm{C}(1)$ & $63(2)$ & $43(2)$ & $45(2)$ & $-7(2)$ & $-10(2)$ & $-15(2)$ \\
\hline$C(5)$ & $64(2)$ & 74(3) & $28(2)$ & $3(2)$ & $10(2)$ & $-1(2)$ \\
\hline$C(6)$ & $72(3)$ & $60(3)$ & $37(2)$ & $11(2)$ & $3(2)$ & $6(2)$ \\
\hline$C(11)$ & 68(3) & $56(3)$ & 63(3) & $17(2)$ & $6(2)$ & $-27(2)$ \\
\hline $\mathrm{C}(2)$ & $93(3)$ & $33(2)$ & $52(3)$ & $-11(2)$ & $-22(2)$ & $2(2)$ \\
\hline$C(3)$ & $75(3)$ & $58(3)$ & $40(2)$ & $-19(2)$ & $-1(2)$ & $18(2)$ \\
\hline$C(10)$ & $82(3)$ & $81(3)$ & $69(3)$ & $13(2)$ & $-9(2)$ & $-57(3)$ \\
\hline$C(25)$ & $48(2)$ & $38(2)$ & $36(2)$ & $1(2)$ & $15(2)$ & $3(2)$ \\
\hline$C(24)$ & $48(2)$ & $39(2)$ & $39(2)$ & $0(2)$ & $13(2)$ & $4(2)$ \\
\hline$C(26)$ & $82(3)$ & 41(3) & $90(4)$ & $11(2)$ & $8(3)$ & $5(2)$ \\
\hline$C(31)$ & $64(3)$ & $58(3)$ & $76(3)$ & $3(2)$ & $11(2)$ & $-17(2)$ \\
\hline$C(27)$ & $52(2)$ & $81(4)$ & $109(4)$ & $16(3)$ & $-3(2)$ & $22(2)$ \\
\hline$C(30)$ & $54(3)$ & $75(4)$ & $162(6)$ & $36(4)$ & $-16(3)$ & $-11(2)$ \\
\hline$C(29)$ & $101(4)$ & $122(5)$ & $96(4)$ & $-36(3)$ & $70(3)$ & $-24(3)$ \\
\hline$C(28)$ & $99(4)$ & $101(4)$ & $90(4)$ & $2(3)$ & $60(3)$ & $29(3)$ \\
\hline$C(16)$ & $49(2)$ & $44(2)$ & $41(2)$ & $-9(2)$ & $18(2)$ & $8(2)$ \\
\hline$C(20)$ & $54(2)$ & $46(2)$ & $36(2)$ & $0(2)$ & $20(2)$ & $13(2)$ \\
\hline$C(19)$ & $78(3)$ & $39(2)$ & $46(2)$ & $0(2)$ & $28(2)$ & $10(2)$ \\
\hline$C(17)$ & $51(2)$ & $51(3)$ & $56(2)$ & $-19(2)$ & $17(2)$ & $3(2)$ \\
\hline$C(18)$ & $70(3)$ & $45(3)$ & $58(3)$ & $-15(2)$ & $36(2)$ & $-4(2)$ \\
\hline$C(21)$ & $55(2)$ & $60(3)$ & $50(2)$ & $-3(2)$ & $-2(2)$ & $14(2)$ \\
\hline$C(23)$ & 71(3) & $66(3)$ & $52(2)$ & $3(2)$ & $3(2)$ & $32(2)$ \\
\hline$C(22)$ & $97(3)$ & $50(3)$ & $106(4)$ & $-14(3)$ & $45(3)$ & $-12(2)$ \\
\hline
\end{tabular}

Stable 8. Bond lengths $[\AA ̊]$ and angles [deg] for complex 2 .

\begin{tabular}{llll}
\hline $\mathrm{Lu}-\mathrm{C}(25)$ & $2.319(3)$ & $\mathrm{Lu}-\mathrm{C}(9)$ & $2.675(3)$ \\
$\mathrm{Lu}-\mathrm{C}(24)$ & $2.319(3)$ & $\mathrm{Si}(2)-\mathrm{C}(25)$ & $1.817(3)$ \\
$\mathrm{Lu}-\mathrm{C}(12)$ & $2.443(3)$ & $\mathrm{Si}(2)-\mathrm{C}(31)$ & $1.873(4)$ \\
$\mathrm{Lu}-\mathrm{C}(3)$ & $2.584(3)$ & $\mathrm{Si}(2)-\mathrm{C}(29)$ & $1.873(4)$ \\
$\mathrm{Lu}-\mathrm{C}(2)$ & $2.607(4)$ & $\mathrm{Si}(2)-\mathrm{C}(30)$ & $1.879(4)$ \\
$\mathrm{Lu}-\mathrm{C}(4)$ & $2.653(3)$ & $\mathrm{Si}(1)-\mathrm{C}(24)$ & $1.832(3)$ \\
$\mathrm{Lu}-\mathrm{C}(1)$ & $2.661(3)$ & $\mathrm{Si}(1)-\mathrm{C}(27)$ & $1.868(4)$
\end{tabular}




\begin{tabular}{|c|c|c|c|}
\hline $\operatorname{Si}(1)-C(28)$ & $1.875(4)$ & $\mathrm{C}(31)-\mathrm{H}(31 \mathrm{C})$ & 0.9600 \\
\hline $\operatorname{Si}(1)-C(26)$ & $1.876(4)$ & $\mathrm{C}(27)-\mathrm{H}(27 \mathrm{~A})$ & 0.9600 \\
\hline $\mathrm{N}(2)-\mathrm{C}(12)$ & $1.357(4)$ & $\mathrm{C}(27)-\mathrm{H}(27 \mathrm{~B})$ & 0.9600 \\
\hline $\mathrm{N}(2)-\mathrm{C}(13)$ & $1.382(4)$ & $\mathrm{C}(27)-\mathrm{H}(27 \mathrm{C})$ & 0.9600 \\
\hline $\mathrm{N}(2)-\mathrm{C}(15)$ & $1.441(4)$ & $\mathrm{C}(30)-\mathrm{H}(30 \mathrm{~A})$ & 0.9600 \\
\hline $\mathrm{N}(1)-\mathrm{C}(12)$ & $1.361(4)$ & $\mathrm{C}(30)-\mathrm{H}(30 \mathrm{~B})$ & 0.9600 \\
\hline $\mathrm{N}(1)-\mathrm{C}(14)$ & $1.376(5)$ & $\mathrm{C}(30)-\mathrm{H}(30 \mathrm{C})$ & 0.9600 \\
\hline $\mathrm{N}(1)-\mathrm{C}(11)$ & $1.458(5)$ & $\mathrm{C}(29)-\mathrm{H}(29 \mathrm{~A})$ & 0.9600 \\
\hline$C(15)-C(20)$ & $1.391(4)$ & $\mathrm{C}(29)-\mathrm{H}(29 \mathrm{~B})$ & 0.9600 \\
\hline$C(15)-C(16)$ & $1.398(4)$ & $\mathrm{C}(29)-\mathrm{H}(29 \mathrm{C})$ & 0.9600 \\
\hline$C(14)-C(13)$ & $1.339(5)$ & $\mathrm{C}(28)-\mathrm{H}(28 \mathrm{~A})$ & 0.9600 \\
\hline $\mathrm{C}(14)-\mathrm{H}(14)$ & 0.9300 & $\mathrm{C}(28)-\mathrm{H}(28 \mathrm{~B})$ & 0.9600 \\
\hline $\mathrm{C}(13)-\mathrm{H}(13)$ & 0.9300 & $\mathrm{C}(28)-\mathrm{H}(28 \mathrm{C})$ & 0.9600 \\
\hline $\mathrm{C}(9)-\mathrm{C}(8)$ & $1.419(5)$ & $C(16)-C(17)$ & $1.390(5)$ \\
\hline $\mathrm{C}(9)-\mathrm{C}(1)$ & $1.427(5)$ & $C(16)-C(21)$ & $1.496(5)$ \\
\hline $\mathrm{C}(9)-\mathrm{C}(4)$ & $1.439(5)$ & $C(20)-C(19)$ & $1.392(5)$ \\
\hline$C(4)-C(3)$ & $1.413(5)$ & $C(20)-C(23)$ & $1.516(5)$ \\
\hline $\mathrm{C}(4)-\mathrm{C}(5)$ & $1.419(5)$ & $\mathrm{C}(19)-\mathrm{C}(18)$ & $1.390(5)$ \\
\hline $\mathrm{C}(8)-\mathrm{C}(7)$ & $1.350(5)$ & $\mathrm{C}(19)-\mathrm{H}(19)$ & 0.9300 \\
\hline $\mathrm{C}(8)-\mathrm{H}(8)$ & 0.9300 & $\mathrm{C}(17)-\mathrm{C}(18)$ & $1.373(5)$ \\
\hline$C(7)-C(6)$ & $1.400(5)$ & $\mathrm{C}(17)-\mathrm{H}(17)$ & 0.9300 \\
\hline $\mathrm{C}(7)-\mathrm{H}(7)$ & 0.9300 & $C(18)-C(22)$ & $1.512(5)$ \\
\hline $\mathrm{C}(1)-\mathrm{C}(2)$ & $1.410(6)$ & $\mathrm{C}(21)-\mathrm{H}(21 \mathrm{~A})$ & 0.9600 \\
\hline C(1)-C(10) & $1.499(5)$ & $\mathrm{C}(21)-\mathrm{H}(21 \mathrm{~B})$ & 0.9600 \\
\hline$C(5)-C(6)$ & $1.351(5)$ & $\mathrm{C}(21)-\mathrm{H}(21 \mathrm{C})$ & 0.9600 \\
\hline $\mathrm{C}(5)-\mathrm{H}(5)$ & 0.9300 & $\mathrm{C}(23)-\mathrm{H}(23 \mathrm{~A})$ & 0.9600 \\
\hline $\mathrm{C}(6)-\mathrm{H}(6)$ & 0.9300 & $\mathrm{C}(23)-\mathrm{H}(23 \mathrm{~B})$ & 0.9600 \\
\hline $\mathrm{C}(11)-\mathrm{C}(10)$ & $1.531(5)$ & $\mathrm{C}(23)-\mathrm{H}(23 \mathrm{C})$ & 0.9600 \\
\hline $\mathrm{C}(11)-\mathrm{H}(11 \mathrm{~A})$ & 0.9700 & $\mathrm{C}(22)-\mathrm{H}(22 \mathrm{~A})$ & 0.9600 \\
\hline $\mathrm{C}(11)-\mathrm{H}(11 \mathrm{~B})$ & 0.9700 & $\mathrm{C}(22)-\mathrm{H}(22 \mathrm{~B})$ & 0.9600 \\
\hline $\mathrm{C}(2)-\mathrm{C}(3)$ & $1.397(6)$ & $\mathrm{C}(22)-\mathrm{H}(22 \mathrm{C})$ & 0.9600 \\
\hline $\mathrm{C}(2)-\mathrm{H}(2)$ & 0.9300 & $\mathrm{C}(25)-\mathrm{Lu}-\mathrm{C}(24)$ & $106.60(12)$ \\
\hline $\mathrm{C}(3)-\mathrm{H}(3)$ & 0.9300 & $\mathrm{C}(25)-\mathrm{Lu}-\mathrm{C}(12)$ & $106.35(11)$ \\
\hline $\mathrm{C}(10)-\mathrm{H}(10 \mathrm{~A})$ & 0.9700 & $\mathrm{C}(24)-\mathrm{Lu}-\mathrm{C}(12)$ & $94.98(11)$ \\
\hline $\mathrm{C}(10)-\mathrm{H}(10 \mathrm{~B})$ & 0.9700 & $\mathrm{C}(25)-\mathrm{Lu}-\mathrm{C}(3)$ & $110.25(13)$ \\
\hline $\mathrm{C}(25)-\mathrm{H}(25 \mathrm{~A})$ & 0.9700 & $\mathrm{C}(24)-\mathrm{Lu}-\mathrm{C}(3)$ & $107.41(12)$ \\
\hline $\mathrm{C}(25)-\mathrm{H}(25 \mathrm{~B})$ & 0.9700 & $\mathrm{C}(12)-\mathrm{Lu}-\mathrm{C}(3)$ & $128.59(13)$ \\
\hline $\mathrm{C}(24)-\mathrm{H}(24 \mathrm{~A})$ & 0.9700 & $\mathrm{C}(25)-\mathrm{Lu}-\mathrm{C}(2)$ & $141.15(13)$ \\
\hline $\mathrm{C}(24)-\mathrm{H}(24 \mathrm{~B})$ & 0.9700 & $\mathrm{C}(24)-\mathrm{Lu}-\mathrm{C}(2)$ & $95.47(12)$ \\
\hline $\mathrm{C}(26)-\mathrm{H}(26 \mathrm{~A})$ & 0.9600 & $\mathrm{C}(12)-\mathrm{Lu}-\mathrm{C}(2)$ & $103.02(14)$ \\
\hline $\mathrm{C}(26)-\mathrm{H}(26 \mathrm{~B})$ & 0.9600 & $\mathrm{C}(3)-\mathrm{Lu}-\mathrm{C}(2)$ & $31.22(13)$ \\
\hline $\mathrm{C}(26)-\mathrm{H}(26 \mathrm{C})$ & 0.9600 & $\mathrm{C}(25)-\mathrm{Lu}-\mathrm{C}(4)$ & $93.12(11)$ \\
\hline $\mathrm{C}(31)-\mathrm{H}(31 \mathrm{~A})$ & 0.9600 & $\mathrm{C}(24)-\mathrm{Lu}-\mathrm{C}(4)$ & $138.59(11)$ \\
\hline $\mathrm{C}(31)-\mathrm{H}(31 \mathrm{~B})$ & 0.9600 & $\mathrm{C}(12)-\mathrm{Lu}-\mathrm{C}(4)$ & $114.23(11)$ \\
\hline
\end{tabular}




\begin{tabular}{|c|c|c|c|}
\hline C(3)-Lu-C(4) & $31.28(10)$ & $\mathrm{N}(2)-\mathrm{C}(13)-\mathrm{H}(13)$ & 126.7 \\
\hline C(2)-Lu-C(4) & $51.22(12)$ & $\mathrm{C}(8)-\mathrm{C}(9)-\mathrm{C}(1)$ & $132.3(4)$ \\
\hline $\mathrm{C}(25)-\mathrm{Lu}-\mathrm{C}(1)$ & $139.32(12)$ & $C(8)-C(9)-C(4)$ & $118.8(3)$ \\
\hline $\mathrm{C}(24)-\mathrm{Lu}-\mathrm{C}(1)$ & $113.63(13)$ & $\mathrm{C}(8)-\mathrm{C}(9)-\mathrm{Lu}$ & $117.7(2)$ \\
\hline $\mathrm{C}(12)-\mathrm{Lu}-\mathrm{C}(1)$ & $76.54(12)$ & $\mathrm{C}(1)-\mathrm{C}(9)-\mathrm{Lu}$ & $73.95(18)$ \\
\hline C(3)-Lu-C(1) & $52.18(13)$ & $\mathrm{C}(4)-\mathrm{C}(9)-\mathrm{Lu}$ & $73.49(18)$ \\
\hline $\mathrm{C}(2)-\mathrm{Lu}-\mathrm{C}(1)$ & $31.03(12)$ & $C(3)-C(4)-C(5)$ & $134.0(4)$ \\
\hline $\mathrm{C}(4)-\mathrm{Lu}-\mathrm{C}(1)$ & $52.04(12)$ & $C(3)-C(4)-C(9)$ & $106.8(3)$ \\
\hline $\mathrm{C}(25)-\mathrm{Lu}-\mathrm{C}(9)$ & $108.29(11)$ & $\mathrm{C}(5)-\mathrm{C}(4)-\mathrm{C}(9)$ & $119.2(3)$ \\
\hline $\mathrm{C}(24)-\mathrm{Lu}-\mathrm{C}(9)$ & $144.04(11)$ & $\mathrm{C}(3)-\mathrm{C}(4)-\mathrm{Lu}$ & $71.66(19)$ \\
\hline $\mathrm{C}(12)-\mathrm{Lu}-\mathrm{C}(9)$ & $83.46(11)$ & $C(5)-C(4)-L u$ & $118.5(2)$ \\
\hline $\mathrm{C}(3)-\mathrm{Lu}-\mathrm{C}(9)$ & $51.58(12)$ & $C(9)-C(4)-L u$ & $75.18(18)$ \\
\hline $\mathrm{C}(2)-\mathrm{Lu}-\mathrm{C}(9)$ & $50.69(11)$ & $\mathrm{C}(7)-\mathrm{C}(8)-\mathrm{C}(9)$ & 119.4(4) \\
\hline $\mathrm{C}(4)-\mathrm{Lu}-\mathrm{C}(9)$ & $31.33(11)$ & $\mathrm{C}(7)-\mathrm{C}(8)-\mathrm{H}(8)$ & 120.3 \\
\hline C(1)-Lu-C(9) & $31.03(10)$ & $\mathrm{C}(9)-\mathrm{C}(8)-\mathrm{H}(8)$ & 120.3 \\
\hline $\mathrm{C}(25)-\mathrm{Si}(2)-\mathrm{C}(31)$ & 114.61(17) & $C(8)-C(7)-C(6)$ & $121.7(4)$ \\
\hline$C(25)-\operatorname{Si}(2)-C(29)$ & $110.07(18)$ & $\mathrm{C}(8)-\mathrm{C}(7)-\mathrm{H}(7)$ & 119.2 \\
\hline $\mathrm{C}(31)-\mathrm{Si}(2)-\mathrm{C}(29)$ & $107.5(2)$ & $\mathrm{C}(6)-\mathrm{C}(7)-\mathrm{H}(7)$ & 119.2 \\
\hline$C(25)-\operatorname{Si}(2)-C(30)$ & $111.66(19)$ & $\mathrm{C}(2)-\mathrm{C}(1)-\mathrm{C}(9)$ & $105.7(4)$ \\
\hline $\mathrm{C}(31)-\mathrm{Si}(2)-\mathrm{C}(30)$ & $105.0(2)$ & $C(2)-C(1)-C(10)$ & $127.1(4)$ \\
\hline$C(29)-\operatorname{Si}(2)-C(30)$ & $107.6(3)$ & $C(9)-C(1)-C(10)$ & $127.1(4)$ \\
\hline $\mathrm{C}(24)-\mathrm{Si}(1)-\mathrm{C}(27)$ & $113.13(19)$ & $C(2)-C(1)-L u$ & $72.3(2)$ \\
\hline $\mathrm{C}(24)-\mathrm{Si}(1)-\mathrm{C}(28)$ & $112.31(19)$ & $\mathrm{C}(9)-\mathrm{C}(1)-\mathrm{Lu}$ & $75.03(19)$ \\
\hline $\mathrm{C}(27)-\mathrm{Si}(1)-\mathrm{C}(28)$ & $106.7(2)$ & $\mathrm{C}(10)-\mathrm{C}(1)-\mathrm{Lu}$ & $120.2(2)$ \\
\hline $\mathrm{C}(24)-\mathrm{Si}(1)-\mathrm{C}(26)$ & 109.04(19) & $C(6)-C(5)-C(4)$ & $119.3(4)$ \\
\hline $\mathrm{C}(27)-\mathrm{Si}(1)-\mathrm{C}(26)$ & $108.1(2)$ & $\mathrm{C}(6)-\mathrm{C}(5)-\mathrm{H}(5)$ & 120.3 \\
\hline $\mathrm{C}(28)-\mathrm{Si}(1)-\mathrm{C}(26)$ & $107.4(2)$ & $\mathrm{C}(4)-\mathrm{C}(5)-\mathrm{H}(5)$ & 120.3 \\
\hline $\mathrm{C}(12)-\mathrm{N}(2)-\mathrm{C}(13)$ & $111.5(3)$ & $C(5)-C(6)-C(7)$ & $121.5(4)$ \\
\hline $\mathrm{C}(12)-\mathrm{N}(2)-\mathrm{C}(15)$ & $124.0(3)$ & $\mathrm{C}(5)-\mathrm{C}(6)-\mathrm{H}(6)$ & 119.3 \\
\hline $\mathrm{C}(13)-\mathrm{N}(2)-\mathrm{C}(15)$ & $124.4(3)$ & $\mathrm{C}(7)-\mathrm{C}(6)-\mathrm{H}(6)$ & 119.3 \\
\hline $\mathrm{C}(12)-\mathrm{N}(1)-\mathrm{C}(14)$ & $111.4(3)$ & $\mathrm{N}(1)-\mathrm{C}(11)-\mathrm{C}(10)$ & $112.7(3)$ \\
\hline $\mathrm{C}(12)-\mathrm{N}(1)-\mathrm{C}(11)$ & $124.3(3)$ & $\mathrm{N}(1)-\mathrm{C}(11)-\mathrm{H}(11 \mathrm{~A})$ & 109.0 \\
\hline $\mathrm{C}(14)-\mathrm{N}(1)-\mathrm{C}(11)$ & $124.3(3)$ & $\mathrm{C}(10)-\mathrm{C}(11)-\mathrm{H}(11 \mathrm{~A})$ & 109.0 \\
\hline$C(20)-C(15)-C(16)$ & $123.0(3)$ & N(1)-C(11)-H(11B) & 109.0 \\
\hline $\mathrm{C}(20)-\mathrm{C}(15)-\mathrm{N}(2)$ & $119.4(3)$ & $\mathrm{C}(10)-\mathrm{C}(11)-\mathrm{H}(11 \mathrm{~B})$ & 109.0 \\
\hline $\mathrm{C}(16)-\mathrm{C}(15)-\mathrm{N}(2)$ & $117.6(3)$ & $\mathrm{H}(11 \mathrm{~A})-\mathrm{C}(11)-\mathrm{H}(11 \mathrm{~B})$ & 107.8 \\
\hline $\mathrm{C}(13)-\mathrm{C}(14)-\mathrm{N}(1)$ & $107.0(3)$ & $\mathrm{C}(3)-\mathrm{C}(2)-\mathrm{C}(1)$ & $110.6(4)$ \\
\hline $\mathrm{C}(13)-\mathrm{C}(14)-\mathrm{H}(14)$ & 126.5 & $\mathrm{C}(3)-\mathrm{C}(2)-\mathrm{Lu}$ & $73.5(2)$ \\
\hline $\mathrm{N}(1)-\mathrm{C}(14)-\mathrm{H}(14)$ & 126.5 & $\mathrm{C}(1)-\mathrm{C}(2)-\mathrm{Lu}$ & $76.6(2)$ \\
\hline $\mathrm{N}(2)-\mathrm{C}(12)-\mathrm{N}(1)$ & $103.5(3)$ & $\mathrm{C}(3)-\mathrm{C}(2)-\mathrm{H}(2)$ & 124.7 \\
\hline $\mathrm{N}(2)-\mathrm{C}(12)-\mathrm{Lu}$ & $132.1(2)$ & $\mathrm{C}(1)-\mathrm{C}(2)-\mathrm{H}(2)$ & 124.7 \\
\hline $\mathrm{N}(1)-\mathrm{C}(12)-\mathrm{Lu}$ & $124.3(3)$ & $\mathrm{Lu}-\mathrm{C}(2)-\mathrm{H}(2)$ & 116.9 \\
\hline $\mathrm{C}(14)-\mathrm{C}(13)-\mathrm{N}(2)$ & $106.5(4)$ & $\mathrm{C}(2)-\mathrm{C}(3)-\mathrm{C}(4)$ & $108.0(4)$ \\
\hline $\mathrm{C}(14)-\mathrm{C}(13)-\mathrm{H}(13)$ & 126.7 & $\mathrm{C}(2)-\mathrm{C}(3)-\mathrm{Lu}$ & $75.3(2)$ \\
\hline
\end{tabular}




\begin{tabular}{|c|c|c|c|}
\hline $\mathrm{C}(4)-\mathrm{C}(3)-\mathrm{Lu}$ & $77.1(2)$ & $\mathrm{H}(30 \mathrm{~A})-\mathrm{C}(30)-\mathrm{H}(30 \mathrm{C})$ & 109.5 \\
\hline $\mathrm{C}(2)-\mathrm{C}(3)-\mathrm{H}(3)$ & 126.0 & $\mathrm{H}(30 \mathrm{~B})-\mathrm{C}(30)-\mathrm{H}(30 \mathrm{C})$ & 109.5 \\
\hline $\mathrm{C}(4)-\mathrm{C}(3)-\mathrm{H}(3)$ & 126.0 & $\mathrm{Si}(2)-\mathrm{C}(29)-\mathrm{H}(29 \mathrm{~A})$ & 109.5 \\
\hline Lu-C(3)-H(3) & 114.0 & $\mathrm{Si}(2)-\mathrm{C}(29)-\mathrm{H}(29 \mathrm{~B})$ & 109.5 \\
\hline $\mathrm{C}(1)-\mathrm{C}(10)-\mathrm{C}(11)$ & $115.5(3)$ & $\mathrm{H}(29 \mathrm{~A})-\mathrm{C}(29)-\mathrm{H}(29 \mathrm{~B})$ & 109.5 \\
\hline $\mathrm{C}(1)-\mathrm{C}(10)-\mathrm{H}(10 \mathrm{~A})$ & 108.4 & $\mathrm{Si}(2)-\mathrm{C}(29)-\mathrm{H}(29 \mathrm{C})$ & 109.5 \\
\hline $\mathrm{C}(11)-\mathrm{C}(10)-\mathrm{H}(10 \mathrm{~A})$ & 108.4 & $\mathrm{H}(29 \mathrm{~A})-\mathrm{C}(29)-\mathrm{H}(29 \mathrm{C})$ & 109.5 \\
\hline $\mathrm{C}(1)-\mathrm{C}(10)-\mathrm{H}(10 \mathrm{~B})$ & 108.4 & $\mathrm{H}(29 \mathrm{~B})-\mathrm{C}(29)-\mathrm{H}(29 \mathrm{C})$ & 109.5 \\
\hline C(11)-C(10)-H(10B) & 108.4 & $\mathrm{Si}(1)-\mathrm{C}(28)-\mathrm{H}(28 \mathrm{~A})$ & 109.5 \\
\hline $\mathrm{H}(10 \mathrm{~A})-\mathrm{C}(10)-\mathrm{H}(10 \mathrm{~B})$ & 107.5 & $\mathrm{Si}(1)-\mathrm{C}(28)-\mathrm{H}(28 \mathrm{~B})$ & 109.5 \\
\hline $\mathrm{Si}(2)-\mathrm{C}(25)-\mathrm{Lu}$ & $129.27(16)$ & $\mathrm{H}(28 \mathrm{~A})-\mathrm{C}(28)-\mathrm{H}(28 \mathrm{~B})$ & 109.5 \\
\hline $\mathrm{Si}(2)-\mathrm{C}(25)-\mathrm{H}(25 \mathrm{~A})$ & 105.0 & $\mathrm{Si}(1)-\mathrm{C}(28)-\mathrm{H}(28 \mathrm{C})$ & 109.5 \\
\hline $\mathrm{Lu}-\mathrm{C}(25)-\mathrm{H}(25 \mathrm{~A})$ & 105.0 & $\mathrm{H}(28 \mathrm{~A})-\mathrm{C}(28)-\mathrm{H}(28 \mathrm{C})$ & 109.5 \\
\hline $\mathrm{Si}(2)-\mathrm{C}(25)-\mathrm{H}(25 \mathrm{~B})$ & 105.0 & $\mathrm{H}(28 \mathrm{~B})-\mathrm{C}(28)-\mathrm{H}(28 \mathrm{C})$ & 109.5 \\
\hline Lu-C(25)-H(25B) & 105.0 & $C(17)-C(16)-C(15)$ & $116.7(3)$ \\
\hline $\mathrm{H}(25 \mathrm{~A})-\mathrm{C}(25)-\mathrm{H}(25 \mathrm{~B})$ & 105.8 & $C(17)-C(16)-C(21)$ & $121.5(3)$ \\
\hline $\mathrm{Si}(1)-\mathrm{C}(24)-\mathrm{Lu}$ & $129.36(17)$ & $\mathrm{C}(15)-\mathrm{C}(16)-\mathrm{C}(21)$ & $121.8(3)$ \\
\hline $\mathrm{Si}(1)-\mathrm{C}(24)-\mathrm{H}(24 \mathrm{~A})$ & 104.9 & $C(15)-C(20)-C(19)$ & $117.0(3)$ \\
\hline $\mathrm{Lu}-\mathrm{C}(24)-\mathrm{H}(24 \mathrm{~A})$ & 104.9 & $C(15)-C(20)-C(23)$ & $120.6(3)$ \\
\hline $\mathrm{Si}(1)-\mathrm{C}(24)-\mathrm{H}(24 \mathrm{~B})$ & 104.9 & $\mathrm{C}(19)-\mathrm{C}(20)-\mathrm{C}(23)$ & $122.4(3)$ \\
\hline $\mathrm{Lu}-\mathrm{C}(24)-\mathrm{H}(24 \mathrm{~B})$ & 104.9 & $\mathrm{C}(18)-\mathrm{C}(19)-\mathrm{C}(20)$ & $122.2(4)$ \\
\hline $\mathrm{H}(24 \mathrm{~A})-\mathrm{C}(24)-\mathrm{H}(24 \mathrm{~B})$ & 105.8 & $\mathrm{C}(18)-\mathrm{C}(19)-\mathrm{H}(19)$ & 118.9 \\
\hline $\mathrm{Si}(1)-\mathrm{C}(26)-\mathrm{H}(26 \mathrm{~A})$ & 109.5 & $\mathrm{C}(20)-\mathrm{C}(19)-\mathrm{H}(19)$ & 118.9 \\
\hline $\mathrm{Si}(1)-\mathrm{C}(26)-\mathrm{H}(26 \mathrm{~B})$ & 109.5 & $\mathrm{C}(18)-\mathrm{C}(17)-\mathrm{C}(16)$ & $122.8(4)$ \\
\hline $\mathrm{H}(26 \mathrm{~A})-\mathrm{C}(26)-\mathrm{H}(26 \mathrm{~B})$ & 109.5 & $\mathrm{C}(18)-\mathrm{C}(17)-\mathrm{H}(17)$ & 118.6 \\
\hline $\mathrm{Si}(1)-\mathrm{C}(26)-\mathrm{H}(26 \mathrm{C})$ & 109.5 & $\mathrm{C}(16)-\mathrm{C}(17)-\mathrm{H}(17)$ & 118.6 \\
\hline $\mathrm{H}(26 \mathrm{~A})-\mathrm{C}(26)-\mathrm{H}(26 \mathrm{C})$ & 109.5 & $\mathrm{C}(17)-\mathrm{C}(18)-\mathrm{C}(19)$ & $118.2(4)$ \\
\hline $\mathrm{H}(26 \mathrm{~B})-\mathrm{C}(26)-\mathrm{H}(26 \mathrm{C})$ & 109.5 & $\mathrm{C}(17)-\mathrm{C}(18)-\mathrm{C}(22)$ & $121.9(4)$ \\
\hline $\mathrm{Si}(2)-\mathrm{C}(31)-\mathrm{H}(31 \mathrm{~A})$ & 109.5 & $\mathrm{C}(19)-\mathrm{C}(18)-\mathrm{C}(22)$ & $119.9(4)$ \\
\hline $\mathrm{Si}(2)-\mathrm{C}(31)-\mathrm{H}(31 \mathrm{~B})$ & 109.5 & $\mathrm{C}(16)-\mathrm{C}(21)-\mathrm{H}(21 \mathrm{~A})$ & 109.5 \\
\hline $\mathrm{H}(31 \mathrm{~A})-\mathrm{C}(31)-\mathrm{H}(31 \mathrm{~B})$ & 109.5 & $\mathrm{C}(16)-\mathrm{C}(21)-\mathrm{H}(21 \mathrm{~B})$ & 109.5 \\
\hline $\mathrm{Si}(2)-\mathrm{C}(31)-\mathrm{H}(31 \mathrm{C})$ & 109.5 & $\mathrm{H}(21 \mathrm{~A})-\mathrm{C}(21)-\mathrm{H}(21 \mathrm{~B})$ & 109.5 \\
\hline $\mathrm{H}(31 \mathrm{~A})-\mathrm{C}(31)-\mathrm{H}(31 \mathrm{C})$ & 109.5 & $\mathrm{C}(16)-\mathrm{C}(21)-\mathrm{H}(21 \mathrm{C})$ & 109.5 \\
\hline $\mathrm{H}(31 \mathrm{~B})-\mathrm{C}(31)-\mathrm{H}(31 \mathrm{C})$ & 109.5 & $\mathrm{H}(21 \mathrm{~A})-\mathrm{C}(21)-\mathrm{H}(21 \mathrm{C})$ & 109.5 \\
\hline $\mathrm{Si}(1)-\mathrm{C}(27)-\mathrm{H}(27 \mathrm{~A})$ & 109.5 & $\mathrm{H}(21 \mathrm{~B})-\mathrm{C}(21)-\mathrm{H}(21 \mathrm{C})$ & 109.5 \\
\hline $\mathrm{Si}(1)-\mathrm{C}(27)-\mathrm{H}(27 \mathrm{~B})$ & 109.5 & $\mathrm{C}(20)-\mathrm{C}(23)-\mathrm{H}(23 \mathrm{~A})$ & 109.5 \\
\hline $\mathrm{H}(27 \mathrm{~A})-\mathrm{C}(27)-\mathrm{H}(27 \mathrm{~B})$ & 109.5 & $\mathrm{C}(20)-\mathrm{C}(23)-\mathrm{H}(23 \mathrm{~B})$ & 109.5 \\
\hline $\mathrm{Si}(1)-\mathrm{C}(27)-\mathrm{H}(27 \mathrm{C})$ & 109.5 & $\mathrm{H}(23 \mathrm{~A})-\mathrm{C}(23)-\mathrm{H}(23 \mathrm{~B})$ & 109.5 \\
\hline $\mathrm{H}(27 \mathrm{~A})-\mathrm{C}(27)-\mathrm{H}(27 \mathrm{C})$ & 109.5 & $\mathrm{C}(20)-\mathrm{C}(23)-\mathrm{H}(23 \mathrm{C})$ & 109.5 \\
\hline $\mathrm{H}(27 \mathrm{~B})-\mathrm{C}(27)-\mathrm{H}(27 \mathrm{C})$ & 109.5 & $\mathrm{H}(23 \mathrm{~A})-\mathrm{C}(23)-\mathrm{H}(23 \mathrm{C})$ & 109.5 \\
\hline $\mathrm{Si}(2)-\mathrm{C}(30)-\mathrm{H}(30 \mathrm{~A})$ & 109.5 & $\mathrm{H}(23 \mathrm{~B})-\mathrm{C}(23)-\mathrm{H}(23 \mathrm{C})$ & 109.5 \\
\hline $\mathrm{Si}(2)-\mathrm{C}(30)-\mathrm{H}(30 \mathrm{~B})$ & 109.5 & $\mathrm{C}(18)-\mathrm{C}(22)-\mathrm{H}(22 \mathrm{~A})$ & 109.5 \\
\hline $\mathrm{H}(30 \mathrm{~A})-\mathrm{C}(30)-\mathrm{H}(30 \mathrm{~B})$ & 109.5 & $\mathrm{C}(18)-\mathrm{C}(22)-\mathrm{H}(22 \mathrm{~B})$ & 109.5 \\
\hline $\mathrm{Si}(2)-\mathrm{C}(30)-\mathrm{H}(30 \mathrm{C})$ & 109.5 & $\mathrm{H}(22 \mathrm{~A})-\mathrm{C}(22)-\mathrm{H}(22 \mathrm{~B})$ & 109.5 \\
\hline
\end{tabular}


$\mathrm{C}(18)-\mathrm{C}(22)-\mathrm{H}(22 \mathrm{C}) \quad 109.5$

Stable 9. Crystal data and structure refinement for 3

\begin{tabular}{|c|c|c|}
\hline Identification code & \multicolumn{2}{|l|}{$\mathrm{r} 081 \mathrm{~m}$} \\
\hline Empirical formula & \multicolumn{2}{|l|}{ C31 H45 N2 Sc Si2 } \\
\hline Formula weight & \multicolumn{2}{|l|}{546.83} \\
\hline Temperature & \multicolumn{2}{|l|}{$187(2) \mathrm{K}$} \\
\hline Wavelength & \multicolumn{2}{|l|}{$0.71073 \AA$} \\
\hline Crystal system & \multicolumn{2}{|l|}{ Monoclinic } \\
\hline Space group & \multicolumn{2}{|l|}{$\mathrm{P} 22_{1} / \mathrm{c}$} \\
\hline \multirow[t]{3}{*}{ Unit cell dimensions } & $\mathrm{a}=10.3718(10) \AA$ & $\alpha=90^{\circ}$ \\
\hline & $\mathrm{b}=23.321(2) \AA$ & $\beta=106.253(2)^{\circ}$. \\
\hline & $\mathrm{c}=13.6852(13) \AA$ & $\gamma=90^{\circ}$ \\
\hline Volume & \multicolumn{2}{|l|}{$3177.9(5) \AA^{3}$} \\
\hline $\mathrm{Z}$ & \multicolumn{2}{|l|}{4} \\
\hline Density (calculated) & \multicolumn{2}{|l|}{$1.143 \mathrm{Mg} / \mathrm{m}^{3}$} \\
\hline Absorption coefficient & \multicolumn{2}{|l|}{$0.328 \mathrm{~mm}^{-1}$} \\
\hline $\mathrm{F}(000)$ & \multicolumn{2}{|l|}{1176} \\
\hline Crystal size & \multicolumn{2}{|c|}{$0.24 \times 0.14 \times 0.08 \mathrm{~mm}^{3}$} \\
\hline Theta range for data collection & \multicolumn{2}{|c|}{1.75 to $26.07^{\circ}$} \\
\hline Index ranges & \multicolumn{2}{|c|}{$-12<=\mathrm{h}<=12,-18<=\mathrm{k}<=28,-16<=1<=16$} \\
\hline Reflections collected & \multicolumn{2}{|l|}{17765} \\
\hline Independent reflections & \multicolumn{2}{|c|}{$6277[\mathrm{R}(\mathrm{int})=0.0701]$} \\
\hline Completeness to theta $=27.05^{\circ}$ & \multicolumn{2}{|c|}{$99.7 \%$} \\
\hline Absorption correction & \multicolumn{2}{|c|}{ Semi-empirical from equivalents } \\
\hline Max. and min. transmission & \multicolumn{2}{|c|}{0.9746 and 0.9246} \\
\hline Refinement method & \multicolumn{2}{|c|}{ Full-matrix least-squares on $\mathrm{F}^{2}$} \\
\hline Data / restraints / parameters & \multicolumn{2}{|l|}{$6277 / 0 / 325$} \\
\hline Goodness-of-fit on $\mathrm{F}^{2}$ & \multicolumn{2}{|l|}{0.852} \\
\hline Final $R$ indices $[I>2 \operatorname{sigma}(I)]$ & \multicolumn{2}{|c|}{$\mathrm{R} 1=0.0518, \mathrm{wR} 2=0.0845$} \\
\hline $\mathrm{R}$ indices (all data) & \multicolumn{2}{|c|}{$\mathrm{R} 1=0.1041, \mathrm{wR} 2=0.0986$} \\
\hline Largest diff. peak and hole & \multicolumn{2}{|c|}{0.348 and $-0.262 \mathrm{e}^{-3}$} \\
\hline
\end{tabular}


Stable 10. Atomic coordinates ( $\times \mathbf{1 0}^{4}$ ) and equivalent isotropic displacement parameters $\left(\AA^{2} \times 10^{3}\right)$ for 3. $U(e q)$ is defined as one third of the trace of the orthogonalized $U^{i j}$ tensor.

\begin{tabular}{|c|c|c|c|c|}
\hline & $\mathrm{x}$ & $\mathrm{y}$ & $\mathrm{z}$ & $\mathrm{U}(\mathrm{eq})$ \\
\hline $\mathrm{Sc}$ & $3394(1)$ & $6596(1)$ & 7918(1) & $33(1)$ \\
\hline $\operatorname{Si}(1)$ & $874(1)$ & $7729(1)$ & 6953(1) & $48(1)$ \\
\hline $\operatorname{Si}(2)$ & 771(1) & $5603(1)$ & $8180(1)$ & $42(1)$ \\
\hline $\mathrm{N}(1)$ & $5546(2)$ & $6845(1)$ & $6587(2)$ & $44(1)$ \\
\hline $\mathrm{N}(2)$ & $4881(2)$ & $5987(1)$ & $6228(2)$ & $37(1)$ \\
\hline$C(1)$ & $5462(4)$ & $7176(1)$ & $8780(2)$ & $53(1)$ \\
\hline$C(2)$ & 4354(4) & $7440(1)$ & $8986(3)$ & $63(1)$ \\
\hline$C(3)$ & $3805(3)$ & 7086(1) & $9587(2)$ & $55(1)$ \\
\hline $\mathrm{C}(4)$ & $4629(3)$ & 6593(1) & $9831(2)$ & $43(1)$ \\
\hline$C(5)$ & 4612(3) & $6109(1)$ & $10445(2)$ & $52(1)$ \\
\hline$C(6)$ & $5568(4)$ & $5700(1)$ & $10532(2)$ & $57(1)$ \\
\hline$C(7)$ & $6552(3)$ & $5747(2)$ & 10011(3) & $60(1)$ \\
\hline $\mathrm{C}(8)$ & $6602(3)$ & $6204(2)$ & 9413(2) & $52(1)$ \\
\hline$C(9)$ & $5651(3)$ & $6649(1)$ & $9317(2)$ & $41(1)$ \\
\hline$C(10)$ & $6330(4)$ & $7420(2)$ & $8177(3)$ & $81(1)$ \\
\hline$C(11)$ & 5713(3) & $7422(1)$ & 7023(3) & 64(1) \\
\hline$C(12)$ & 4734(3) & $6440(1)$ & $6814(2)$ & $35(1)$ \\
\hline$C(13)$ & 5751(3) & $6113(2)$ & $5658(2)$ & $56(1)$ \\
\hline$C(14)$ & 6161(3) & $6650(2)$ & $5888(3)$ & $58(1)$ \\
\hline$C(15)$ & 4133(3) & $5457(1)$ & 6132(2) & $36(1)$ \\
\hline$C(16)$ & 2870(3) & $5440(1)$ & $5441(2)$ & $40(1)$ \\
\hline$C(17)$ & 2131(3) & $4940(1)$ & 5402(2) & $48(1)$ \\
\hline$C(18)$ & 2610(3) & $4476(1)$ & 6024(3) & $50(1)$ \\
\hline$C(19)$ & 3892(3) & $4506(1)$ & $6679(2)$ & $49(1)$ \\
\hline$C(20)$ & 4692(3) & 4990(1) & $6745(2)$ & $40(1)$ \\
\hline $\mathrm{C}(21)$ & 6098(3) & $5015(1)$ & $7448(2)$ & $60(1)$ \\
\hline$C(22)$ & $1765(3)$ & $3944(1)$ & 5988(3) & $75(1)$ \\
\hline$C(23)$ & 2316(3) & $5939(1)$ & $4758(2)$ & $58(1)$ \\
\hline$C(24)$ & $1888(3)$ & $7112(1)$ & 6801(2) & $40(1)$ \\
\hline$C(25)$ & $2438(3)$ & $5763(1)$ & $8038(2)$ & $37(1)$ \\
\hline$C(26)$ & $326(4)$ & $7688(2)$ & $8147(3)$ & $83(1)$ \\
\hline$C(27)$ & $1869(3)$ & $8404(1)$ & 7017(3) & $67(1)$ \\
\hline$C(28)$ & $-685(3)$ & 7805(2) & $5870(3)$ & $84(1)$ \\
\hline$C(29)$ & 692(4) & $5775(2)$ & 9491(3) & $84(1)$ \\
\hline $\mathrm{C}(30)$ & $-562(3)$ & 6028(2) & $7265(3)$ & $97(2)$ \\
\hline $\mathrm{C}(31)$ & $258(3)$ & $4836(1)$ & 7921(2) & 61(1) \\
\hline
\end{tabular}


Stable 11. Anisotropic displacement parameters $\left(\AA^{2} x 0^{3}\right)$ for 3. The anisotropic displacement factor exponent takes the form: $-2 \pi^{2}\left[h^{2} a^{* 2} U^{11}+\ldots+2 h k a^{*} b^{*} U^{12}\right]$

\begin{tabular}{|c|c|c|c|c|c|c|}
\hline & U11 & U22 & U33 & U23 & U13 & U12 \\
\hline $\mathrm{Sc}$ & $36(1)$ & $30(1)$ & $32(1)$ & $-2(1)$ & $6(1)$ & $0(1)$ \\
\hline $\operatorname{Si}(1)$ & $46(1)$ & $42(1)$ & $56(1)$ & $9(1)$ & $16(1)$ & $10(1)$ \\
\hline $\operatorname{Si}(2)$ & $37(1)$ & $40(1)$ & $49(1)$ & $-1(1)$ & $10(1)$ & $-5(1)$ \\
\hline $\mathrm{N}(1)$ & $34(2)$ & $50(2)$ & $47(2)$ & $15(1)$ & $7(1)$ & $-7(1)$ \\
\hline $\mathrm{N}(2)$ & $32(1)$ & $46(2)$ & $38(2)$ & $9(1)$ & $15(1)$ & $4(1)$ \\
\hline$C(1)$ & $63(2)$ & $43(2)$ & $42(2)$ & $-3(2)$ & $-4(2)$ & $-20(2)$ \\
\hline$C(2)$ & $90(3)$ & $25(2)$ & $50(2)$ & $-10(2)$ & $-17(2)$ & $3(2)$ \\
\hline$C(3)$ & $65(2)$ & $56(2)$ & $36(2)$ & $-10(2)$ & $3(2)$ & $13(2)$ \\
\hline$C(4)$ & $50(2)$ & $41(2)$ & $32(2)$ & $-9(2)$ & $0(2)$ & $8(2)$ \\
\hline$C(5)$ & $60(2)$ & $62(2)$ & $30(2)$ & $2(2)$ & $7(2)$ & $5(2)$ \\
\hline$C(6)$ & $76(3)$ & $47(2)$ & $39(2)$ & $8(2)$ & $0(2)$ & $8(2)$ \\
\hline$C(7)$ & $52(2)$ & $61(3)$ & $56(2)$ & $-1(2)$ & $-4(2)$ & $17(2)$ \\
\hline$C(8)$ & $36(2)$ & $65(3)$ & $46(2)$ & $-5(2)$ & $-3(2)$ & $-5(2)$ \\
\hline$C(9)$ & $38(2)$ & $39(2)$ & $36(2)$ & $-7(2)$ & $-5(1)$ & $-5(2)$ \\
\hline$C(10)$ & $84(3)$ & $66(3)$ & $72(3)$ & $9(2)$ & $-9(2)$ & $-47(2)$ \\
\hline$C(11)$ & $68(2)$ & $55(3)$ & $64(3)$ & $15(2)$ & $10(2)$ & $-29(2)$ \\
\hline$C(12)$ & $28(2)$ & $38(2)$ & $35(2)$ & $8(1)$ & $2(1)$ & $-1(1)$ \\
\hline$C(13)$ & $50(2)$ & 74(3) & $55(2)$ & $14(2)$ & $33(2)$ & $14(2)$ \\
\hline$C(14)$ & $36(2)$ & $82(3)$ & $60(2)$ & $26(2)$ & $22(2)$ & $0(2)$ \\
\hline$C(15)$ & $38(2)$ & 41(2) & $33(2)$ & $-4(1)$ & $18(2)$ & $5(2)$ \\
\hline$C(16)$ & $44(2)$ & $43(2)$ & $34(2)$ & $-6(2)$ & $12(2)$ & $8(2)$ \\
\hline$C(17)$ & $42(2)$ & $50(2)$ & $50(2)$ & $-14(2)$ & $11(2)$ & $4(2)$ \\
\hline$C(18)$ & $61(2)$ & 41(2) & $54(2)$ & $-16(2)$ & $29(2)$ & $-4(2)$ \\
\hline$C(19)$ & $69(2)$ & $36(2)$ & $45(2)$ & $1(2)$ & $23(2)$ & $12(2)$ \\
\hline$C(20)$ & $48(2)$ & $42(2)$ & $35(2)$ & $-1(2)$ & $17(2)$ & $12(2)$ \\
\hline$C(21)$ & $58(2)$ & $57(2)$ & $57(2)$ & $8(2)$ & $4(2)$ & $17(2)$ \\
\hline$C(22)$ & $81(3)$ & $48(2)$ & $103(3)$ & $-15(2)$ & $39(2)$ & $-7(2)$ \\
\hline$C(23)$ & $53(2)$ & $58(2)$ & $51(2)$ & $0(2)$ & $-2(2)$ & $11(2)$ \\
\hline$C(24)$ & $40(2)$ & $39(2)$ & 41(2) & $2(1)$ & $12(2)$ & $0(1)$ \\
\hline$C(25)$ & $43(2)$ & $35(2)$ & $34(2)$ & $-2(1)$ & $13(1)$ & $0(1)$ \\
\hline$C(26)$ & $95(3)$ & $84(3)$ & 91(3) & $11(2)$ & $59(3)$ & $26(2)$ \\
\hline$C(27)$ & $83(3)$ & $41(2)$ & $73(3)$ & $10(2)$ & $15(2)$ & $11(2)$ \\
\hline$C(28)$ & $53(2)$ & $80(3)$ & $107(3)$ & $17(2)$ & $5(2)$ & $16(2)$ \\
\hline$C(29)$ & $88(3)$ & 94(3) & $89(3)$ & $-27(2)$ & $56(3)$ & $-22(2)$ \\
\hline$C(30)$ & $43(2)$ & $70(3)$ & $154(4)$ & $36(3)$ & $-10(2)$ & $-9(2)$ \\
\hline$C(31)$ & $55(2)$ & $53(2)$ & $69(3)$ & $0(2)$ & $7(2)$ & $-12(2)$ \\
\hline
\end{tabular}


Stable 12. Bond lengths $[\AA]]$ and angles $\left[^{\circ}\right]$ for 3 .

\begin{tabular}{|c|c|c|c|}
\hline $\mathrm{Sc}-\mathrm{C}(25)$ & $2.208(3)$ & $\mathrm{C}(10)-\mathrm{H}(10 \mathrm{~B})$ & 0.9900 \\
\hline $\mathrm{Sc}-\mathrm{C}(24)$ & $2.209(3)$ & $\mathrm{C}(11)-\mathrm{H}(11 \mathrm{~A})$ & 0.9900 \\
\hline Sc-C(12) & $2.350(3)$ & $\mathrm{C}(11)-\mathrm{H}(11 \mathrm{~B})$ & 0.9900 \\
\hline $\mathrm{Sc}-\mathrm{C}(3)$ & $2.482(3)$ & $C(13)-C(14)$ & $1.332(4)$ \\
\hline $\mathrm{Sc}-\mathrm{C}(2)$ & $2.487(3)$ & $\mathrm{C}(13)-\mathrm{H}(13)$ & 0.9500 \\
\hline Sc-C(1) & $2.531(3)$ & $\mathrm{C}(14)-\mathrm{H}(14)$ & 0.9500 \\
\hline Sc-C(4) & $2.572(3)$ & $C(15)-C(16)$ & $1.385(4)$ \\
\hline Sc-C(9) & $2.579(3)$ & $C(15)-C(20)$ & $1.397(4)$ \\
\hline $\operatorname{Si}(1)-C(24)$ & $1.829(3)$ & $C(16)-C(17)$ & $1.388(4)$ \\
\hline $\operatorname{Si}(1)-C(27)$ & $1.870(3)$ & $C(16)-C(23)$ & $1.503(4)$ \\
\hline $\operatorname{Si}(1)-C(28)$ & $1.872(3)$ & $C(17)-C(18)$ & $1.380(4)$ \\
\hline $\operatorname{Si}(1)-C(26)$ & $1.876(3)$ & $\mathrm{C}(17)-\mathrm{H}(17)$ & 0.9500 \\
\hline $\mathrm{Si}(2)-\mathrm{C}(25)$ & $1.831(3)$ & $\mathrm{C}(18)-\mathrm{C}(19)$ & $1.382(4)$ \\
\hline $\operatorname{Si}(2)-C(29)$ & $1.864(3)$ & $\mathrm{C}(18)-\mathrm{C}(22)$ & $1.512(4)$ \\
\hline $\operatorname{Si}(2)-C(30)$ & $1.868(3)$ & C(19)-C(20) & $1.389(4)$ \\
\hline $\mathrm{Si}(2)-\mathrm{C}(31)$ & $1.872(3)$ & $\mathrm{C}(19)-\mathrm{H}(19)$ & 0.9500 \\
\hline $\mathrm{N}(1)-\mathrm{C}(12)$ & $1.358(3)$ & $C(20)-C(21)$ & $1.507(4)$ \\
\hline $\mathrm{N}(1)-\mathrm{C}(14)$ & $1.368(4)$ & $\mathrm{C}(21)-\mathrm{H}(21 \mathrm{~A})$ & 0.9800 \\
\hline $\mathrm{N}(1)-\mathrm{C}(11)$ & $1.464(4)$ & $\mathrm{C}(21)-\mathrm{H}(21 \mathrm{~B})$ & 0.9800 \\
\hline $\mathrm{N}(2)-\mathrm{C}(12)$ & $1.360(3)$ & $\mathrm{C}(21)-\mathrm{H}(21 \mathrm{C})$ & 0.9800 \\
\hline $\mathrm{N}(2)-\mathrm{C}(13)$ & $1.379(3)$ & $\mathrm{C}(22)-\mathrm{H}(22 \mathrm{~A})$ & 0.9800 \\
\hline $\mathrm{N}(2)-\mathrm{C}(15)$ & $1.445(3)$ & $\mathrm{C}(22)-\mathrm{H}(22 \mathrm{~B})$ & 0.9800 \\
\hline$C(1)-C(2)$ & $1.401(4)$ & $\mathrm{C}(22)-\mathrm{H}(22 \mathrm{C})$ & 0.9800 \\
\hline $\mathrm{C}(1)-\mathrm{C}(9)$ & $1.417(4)$ & $\mathrm{C}(23)-\mathrm{H}(23 \mathrm{~A})$ & 0.9800 \\
\hline $\mathrm{C}(1)-\mathrm{C}(10)$ & $1.493(4)$ & $\mathrm{C}(23)-\mathrm{H}(23 \mathrm{~B})$ & 0.9800 \\
\hline$C(2)-C(3)$ & $1.396(4)$ & $\mathrm{C}(23)-\mathrm{H}(23 \mathrm{C})$ & 0.9800 \\
\hline $\mathrm{C}(2)-\mathrm{H}(2)$ & 0.9500 & $\mathrm{C}(24)-\mathrm{H}(24 \mathrm{~A})$ & 0.9900 \\
\hline$C(3)-C(4)$ & $1.416(4)$ & $\mathrm{C}(24)-\mathrm{H}(24 \mathrm{~B})$ & 0.9900 \\
\hline $\mathrm{C}(3)-\mathrm{H}(3)$ & 0.9500 & $\mathrm{C}(25)-\mathrm{H}(25 \mathrm{~A})$ & 0.9900 \\
\hline$C(4)-C(5)$ & $1.410(4)$ & $\mathrm{C}(25)-\mathrm{H}(25 \mathrm{~B})$ & 0.9900 \\
\hline C(4)-C(9) & $1.432(4)$ & $\mathrm{C}(26)-\mathrm{H}(26 \mathrm{~A})$ & 0.9800 \\
\hline$C(5)-C(6)$ & $1.356(4)$ & $\mathrm{C}(26)-\mathrm{H}(26 \mathrm{~B})$ & 0.9800 \\
\hline $\mathrm{C}(5)-\mathrm{H}(5)$ & 0.9500 & $\mathrm{C}(26)-\mathrm{H}(26 \mathrm{C})$ & 0.9800 \\
\hline$C(6)-C(7)$ & $1.403(4)$ & $\mathrm{C}(27)-\mathrm{H}(27 \mathrm{~A})$ & 0.9800 \\
\hline $\mathrm{C}(6)-\mathrm{H}(6)$ & 0.9500 & $\mathrm{C}(27)-\mathrm{H}(27 \mathrm{~B})$ & 0.9800 \\
\hline$C(7)-C(8)$ & $1.353(4)$ & $\mathrm{C}(27)-\mathrm{H}(27 \mathrm{C})$ & 0.9800 \\
\hline $\mathrm{C}(7)-\mathrm{H}(7)$ & 0.9500 & $\mathrm{C}(28)-\mathrm{H}(28 \mathrm{~A})$ & 0.9800 \\
\hline$C(8)-C(9)$ & $1.413(4)$ & $\mathrm{C}(28)-\mathrm{H}(28 \mathrm{~B})$ & 0.9800 \\
\hline $\mathrm{C}(8)-\mathrm{H}(8)$ & 0.9500 & $\mathrm{C}(28)-\mathrm{H}(28 \mathrm{C})$ & 0.9800 \\
\hline$C(10)-C(11)$ & $1.529(4)$ & C(29)-H(29A) & 0.9800 \\
\hline $\mathrm{C}(10)-\mathrm{H}(10 \mathrm{~A})$ & 0.9900 & C(29)-H(29B) & 0.9800 \\
\hline
\end{tabular}




\begin{tabular}{|c|c|c|c|}
\hline $\mathrm{C}(29)-\mathrm{H}(29 \mathrm{C})$ & 0.9800 & $\mathrm{C}(31)-\mathrm{H}(31 \mathrm{~B})$ & 0.9800 \\
\hline $\mathrm{C}(30)-\mathrm{H}(30 \mathrm{~A})$ & 0.9800 & $\mathrm{C}(31)-\mathrm{H}(31 \mathrm{C})$ & 0.9800 \\
\hline $\mathrm{C}(30)-\mathrm{H}(30 \mathrm{~B})$ & 0.9800 & & \\
\hline \multirow[t]{2}{*}{$\mathrm{C}(30)-\mathrm{H}(30 \mathrm{C})$} & 0.9800 & $C(29)-\operatorname{Si}(2)-C(31)$ & $107.48(15)$ \\
\hline & & $C(30)-\operatorname{Si}(2)-C(31)$ & $105.20(15)$ \\
\hline $\mathrm{C}(25)-\mathrm{Sc}-\mathrm{C}(24)$ & $106.56(10)$ & $\mathrm{C}(12)-\mathrm{N}(1)-\mathrm{C}(14)$ & 111.7(3) \\
\hline$C(25)-S c-C(12)$ & $105.67(10)$ & $\mathrm{C}(12)-\mathrm{N}(1)-\mathrm{C}(11)$ & $123.9(3)$ \\
\hline $\mathrm{C}(24)-\mathrm{Sc}-\mathrm{C}(12)$ & $94.04(9)$ & $\mathrm{C}(14)-\mathrm{N}(1)-\mathrm{C}(11)$ & $124.4(3)$ \\
\hline $\mathrm{C}(25)-\mathrm{Sc}-\mathrm{C}(3)$ & $107.50(11)$ & $\mathrm{C}(12)-\mathrm{N}(2)-\mathrm{C}(13)$ & $111.5(3)$ \\
\hline $\mathrm{C}(24)-\mathrm{Sc}-\mathrm{C}(3)$ & 107.01(11) & $\mathrm{C}(12)-\mathrm{N}(2)-\mathrm{C}(15)$ & $124.9(2)$ \\
\hline $\mathrm{C}(12)-\mathrm{Sc}-\mathrm{C}(3)$ & $133.10(11)$ & $\mathrm{C}(13)-\mathrm{N}(2)-\mathrm{C}(15)$ & $123.4(3)$ \\
\hline $\mathrm{C}(25)-\mathrm{Sc}-\mathrm{C}(2)$ & $140.04(12)$ & $\mathrm{C}(2)-\mathrm{C}(1)-\mathrm{C}(9)$ & 106.2(3) \\
\hline $\mathrm{C}(24)-\mathrm{Sc}-\mathrm{C}(2)$ & $93.75(11)$ & $C(2)-C(1)-C(10)$ & $126.8(3)$ \\
\hline $\mathrm{C}(12)-\mathrm{Sc}-\mathrm{C}(2)$ & $106.81(12)$ & $C(9)-C(1)-C(10)$ & $126.9(3)$ \\
\hline $\mathrm{C}(3)-\mathrm{Sc}-\mathrm{C}(2)$ & $32.63(10)$ & $\mathrm{C}(2)-\mathrm{C}(1)-\mathrm{Sc}$ & $72.09(18)$ \\
\hline $\mathrm{C}(25)-\mathrm{Sc}-\mathrm{C}(1)$ & $140.79(11)$ & $\mathrm{C}(9)-\mathrm{C}(1)-\mathrm{Sc}$ & $75.78(17)$ \\
\hline $\mathrm{C}(24)-\mathrm{Sc}-\mathrm{C}(1)$ & $112.01(11)$ & $\mathrm{C}(10)-\mathrm{C}(1)-\mathrm{Sc}$ & $120.6(2)$ \\
\hline $\mathrm{C}(12)-\mathrm{Sc}-\mathrm{C}(1)$ & $78.82(11)$ & $C(3)-C(2)-C(1)$ & $110.6(3)$ \\
\hline $\mathrm{C}(3)-\mathrm{Sc}-\mathrm{C}(1)$ & $54.58(11)$ & $\mathrm{C}(3)-\mathrm{C}(2)-\mathrm{Sc}$ & $73.45(18)$ \\
\hline $\mathrm{C}(2)-\mathrm{Sc}-\mathrm{C}(1)$ & $32.40(10)$ & $\mathrm{C}(1)-\mathrm{C}(2)-\mathrm{Sc}$ & $75.51(18)$ \\
\hline $\mathrm{C}(25)-\mathrm{Sc}-\mathrm{C}(4)$ & $91.56(10)$ & $\mathrm{C}(3)-\mathrm{C}(2)-\mathrm{H}(2)$ & 124.7 \\
\hline C(24)-Sc-C(4) & $139.46(10)$ & $\mathrm{C}(1)-\mathrm{C}(2)-\mathrm{H}(2)$ & 124.7 \\
\hline $\mathrm{C}(12)-\mathrm{Sc}-\mathrm{C}(4)$ & $115.98(10)$ & $\mathrm{Sc}-\mathrm{C}(2)-\mathrm{H}(2)$ & 118.0 \\
\hline $\mathrm{C}(3)-\mathrm{Sc}-\mathrm{C}(4)$ & $32.47(9)$ & $\mathrm{C}(2)-\mathrm{C}(3)-\mathrm{C}(4)$ & $107.5(3)$ \\
\hline C(2)-Sc-C(4) & $53.21(10)$ & $\mathrm{C}(2)-\mathrm{C}(3)-\mathrm{Sc}$ & $73.92(18)$ \\
\hline $\mathrm{C}(1)-\mathrm{Sc}-\mathrm{C}(4)$ & $53.95(10)$ & $\mathrm{C}(4)-\mathrm{C}(3)-\mathrm{Sc}$ & $77.29(17)$ \\
\hline $\mathrm{C}(25)-\mathrm{Sc}-\mathrm{C}(9)$ & $108.65(10)$ & $\mathrm{C}(2)-\mathrm{C}(3)-\mathrm{H}(3)$ & 126.3 \\
\hline $\mathrm{C}(24)-\mathrm{Sc}-\mathrm{C}(9)$ & $143.75(10)$ & $\mathrm{C}(4)-\mathrm{C}(3)-\mathrm{H}(3)$ & 126.3 \\
\hline $\mathrm{C}(12)-\mathrm{Sc}-\mathrm{C}(9)$ & $84.70(10)$ & $\mathrm{Sc}-\mathrm{C}(3)-\mathrm{H}(3)$ & 114.8 \\
\hline $\mathrm{C}(3)-\mathrm{Sc}-\mathrm{C}(9)$ & $53.72(10)$ & $\mathrm{C}(5)-\mathrm{C}(4)-\mathrm{C}(3)$ & $133.0(3)$ \\
\hline $\mathrm{C}(2)-\mathrm{Sc}-\mathrm{C}(9)$ & $52.77(10)$ & $C(5)-C(4)-C(9)$ & $120.0(3)$ \\
\hline $\mathrm{C}(1)-\mathrm{Sc}-\mathrm{C}(9)$ & $32.18(9)$ & $C(3)-C(4)-C(9)$ & $106.9(3)$ \\
\hline C(4)-Sc-C(9) & $32.28(9)$ & $C(5)-C(4)-S c$ & $121.6(2)$ \\
\hline $\mathrm{C}(24)-\mathrm{Si}(1)-\mathrm{C}(27)$ & $109.89(14)$ & $\mathrm{C}(3)-\mathrm{C}(4)-\mathrm{Sc}$ & $70.23(16)$ \\
\hline $\mathrm{C}(24)-\mathrm{Si}(1)-\mathrm{C}(28)$ & $112.45(15)$ & $\mathrm{C}(9)-\mathrm{C}(4)-\mathrm{Sc}$ & $74.12(16)$ \\
\hline $\mathrm{C}(27)-\mathrm{Si}(1)-\mathrm{C}(28)$ & $107.45(15)$ & $C(6)-C(5)-C(4)$ & $119.0(3)$ \\
\hline $\mathrm{C}(24)-\mathrm{Si}(1)-\mathrm{C}(26)$ & $112.52(14)$ & $\mathrm{C}(6)-\mathrm{C}(5)-\mathrm{H}(5)$ & 120.5 \\
\hline$C(27)-\operatorname{Si}(1)-C(26)$ & $107.46(16)$ & $\mathrm{C}(4)-\mathrm{C}(5)-\mathrm{H}(5)$ & 120.5 \\
\hline $\mathrm{C}(28)-\mathrm{Si}(1)-\mathrm{C}(26)$ & $106.81(17)$ & $C(5)-C(6)-C(7)$ & $121.2(3)$ \\
\hline $\mathrm{C}(25)-\mathrm{Si}(2)-\mathrm{C}(29)$ & $110.82(14)$ & $\mathrm{C}(5)-\mathrm{C}(6)-\mathrm{H}(6)$ & 119.4 \\
\hline$C(25)-\operatorname{Si}(2)-C(30)$ & $111.38(14)$ & $\mathrm{C}(7)-\mathrm{C}(6)-\mathrm{H}(6)$ & 119.4 \\
\hline $\mathrm{C}(29)-\mathrm{Si}(2)-\mathrm{C}(30)$ & 107.94(19) & $\mathrm{C}(8)-\mathrm{C}(7)-\mathrm{C}(6)$ & $121.7(3)$ \\
\hline$C(25)-\operatorname{Si}(2)-C(31)$ & $113.69(13)$ & $\mathrm{C}(8)-\mathrm{C}(7)-\mathrm{H}(7)$ & 119.2 \\
\hline $\mathrm{C}(31)-\mathrm{H}(31 \mathrm{~A})$ & 0.9800 & $\mathrm{C}(6)-\mathrm{C}(7)-\mathrm{H}(7)$ & 119.2 \\
\hline
\end{tabular}




\begin{tabular}{|c|c|c|c|}
\hline $\mathrm{C}(7)-\mathrm{C}(8)-\mathrm{C}(9)$ & $119.4(3)$ & $\mathrm{C}(20)-\mathrm{C}(19)-\mathrm{H}(19)$ & 118.9 \\
\hline $\mathrm{C}(7)-\mathrm{C}(8)-\mathrm{H}(8)$ & 120.3 & $C(19)-C(20)-C(15)$ & $117.0(3)$ \\
\hline $\mathrm{C}(9)-\mathrm{C}(8)-\mathrm{H}(8)$ & 120.3 & $C(19)-C(20)-C(21)$ & $121.8(3)$ \\
\hline $\mathrm{C}(8)-\mathrm{C}(9)-\mathrm{C}(1)$ & $132.6(3)$ & $\mathrm{C}(15)-\mathrm{C}(20)-\mathrm{C}(21)$ & $121.2(3)$ \\
\hline $\mathrm{C}(8)-\mathrm{C}(9)-\mathrm{C}(4)$ & $118.7(3)$ & $\mathrm{C}(20)-\mathrm{C}(21)-\mathrm{H}(21 \mathrm{~A})$ & 109.5 \\
\hline $\mathrm{C}(1)-\mathrm{C}(9)-\mathrm{C}(4)$ & $108.7(3)$ & $\mathrm{C}(20)-\mathrm{C}(21)-\mathrm{H}(21 \mathrm{~B})$ & 109.5 \\
\hline $\mathrm{C}(8)-\mathrm{C}(9)-\mathrm{Sc}$ & $119.93(19)$ & $\mathrm{H}(21 \mathrm{~A})-\mathrm{C}(21)-\mathrm{H}(21 \mathrm{~B})$ & 109.5 \\
\hline $\mathrm{C}(1)-\mathrm{C}(9)-\mathrm{Sc}$ & $72.04(17)$ & $\mathrm{C}(20)-\mathrm{C}(21)-\mathrm{H}(21 \mathrm{C})$ & 109.5 \\
\hline $\mathrm{C}(4)-\mathrm{C}(9)-\mathrm{Sc}$ & $73.60(16)$ & $\mathrm{H}(21 \mathrm{~A})-\mathrm{C}(21)-\mathrm{H}(21 \mathrm{C})$ & 109.5 \\
\hline $\mathrm{C}(1)-\mathrm{C}(10)-\mathrm{C}(11)$ & 115.5(3) & $\mathrm{H}(21 \mathrm{~B})-\mathrm{C}(21)-\mathrm{H}(21 \mathrm{C})$ & 109.5 \\
\hline $\mathrm{C}(1)-\mathrm{C}(10)-\mathrm{H}(10 \mathrm{~A})$ & 108.4 & $\mathrm{C}(18)-\mathrm{C}(22)-\mathrm{H}(22 \mathrm{~A})$ & 109.5 \\
\hline $\mathrm{C}(11)-\mathrm{C}(10)-\mathrm{H}(10 \mathrm{~A})$ & 108.4 & $\mathrm{C}(18)-\mathrm{C}(22)-\mathrm{H}(22 \mathrm{~B})$ & 109.5 \\
\hline C(1)-C(10)-H(10B) & 108.4 & $\mathrm{H}(22 \mathrm{~A})-\mathrm{C}(22)-\mathrm{H}(22 \mathrm{~B})$ & 109.5 \\
\hline C(11)-C(10)-H(10B) & 108.4 & $\mathrm{C}(18)-\mathrm{C}(22)-\mathrm{H}(22 \mathrm{C})$ & 109.5 \\
\hline $\mathrm{H}(10 \mathrm{~A})-\mathrm{C}(10)-\mathrm{H}(10 \mathrm{~B})$ & 107.5 & $\mathrm{H}(22 \mathrm{~A})-\mathrm{C}(22)-\mathrm{H}(22 \mathrm{C})$ & 109.5 \\
\hline $\mathrm{N}(1)-\mathrm{C}(11)-\mathrm{C}(10)$ & $112.6(3)$ & $\mathrm{H}(22 \mathrm{~B})-\mathrm{C}(22)-\mathrm{H}(22 \mathrm{C})$ & 109.5 \\
\hline $\mathrm{N}(1)-\mathrm{C}(11)-\mathrm{H}(11 \mathrm{~A})$ & 109.1 & $\mathrm{C}(16)-\mathrm{C}(23)-\mathrm{H}(23 \mathrm{~A})$ & 109.5 \\
\hline $\mathrm{C}(10)-\mathrm{C}(11)-\mathrm{H}(11 \mathrm{~A})$ & 109.1 & $\mathrm{C}(16)-\mathrm{C}(23)-\mathrm{H}(23 \mathrm{~B})$ & 109.5 \\
\hline $\mathrm{N}(1)-\mathrm{C}(11)-\mathrm{H}(11 \mathrm{~B})$ & 109.1 & $\mathrm{H}(23 \mathrm{~A})-\mathrm{C}(23)-\mathrm{H}(23 \mathrm{~B})$ & 109.5 \\
\hline C(10)-C(11)-H(11B) & 109.1 & $\mathrm{C}(16)-\mathrm{C}(23)-\mathrm{H}(23 \mathrm{C})$ & 109.5 \\
\hline $\mathrm{H}(11 \mathrm{~A})-\mathrm{C}(11)-\mathrm{H}(11 \mathrm{~B})$ & 107.8 & $\mathrm{H}(23 \mathrm{~A})-\mathrm{C}(23)-\mathrm{H}(23 \mathrm{C})$ & 109.5 \\
\hline $\mathrm{N}(1)-\mathrm{C}(12)-\mathrm{N}(2)$ & $103.1(2)$ & $\mathrm{H}(23 \mathrm{~B})-\mathrm{C}(23)-\mathrm{H}(23 \mathrm{C})$ & 109.5 \\
\hline $\mathrm{N}(1)-\mathrm{C}(12)-\mathrm{Sc}$ & $123.7(2)$ & $\mathrm{Si}(1)-\mathrm{C}(24)-\mathrm{Sc}$ & $131.85(14)$ \\
\hline $\mathrm{N}(2)-\mathrm{C}(12)-\mathrm{Sc}$ & $133.1(2)$ & $\mathrm{Si}(1)-\mathrm{C}(24)-\mathrm{H}(24 \mathrm{~A})$ & 104.3 \\
\hline $\mathrm{C}(14)-\mathrm{C}(13)-\mathrm{N}(2)$ & $106.5(3)$ & $\mathrm{Sc}-\mathrm{C}(24)-\mathrm{H}(24 \mathrm{~A})$ & 104.3 \\
\hline $\mathrm{C}(14)-\mathrm{C}(13)-\mathrm{H}(13)$ & 126.8 & $\mathrm{Si}(1)-\mathrm{C}(24)-\mathrm{H}(24 \mathrm{~B})$ & 104.3 \\
\hline $\mathrm{N}(2)-\mathrm{C}(13)-\mathrm{H}(13)$ & 126.8 & $\mathrm{Sc}-\mathrm{C}(24)-\mathrm{H}(24 \mathrm{~B})$ & 104.3 \\
\hline $\mathrm{C}(13)-\mathrm{C}(14)-\mathrm{N}(1)$ & $107.2(3)$ & $\mathrm{H}(24 \mathrm{~A})-\mathrm{C}(24)-\mathrm{H}(24 \mathrm{~B})$ & 105.6 \\
\hline $\mathrm{C}(13)-\mathrm{C}(14)-\mathrm{H}(14)$ & 126.4 & $\mathrm{Si}(2)-\mathrm{C}(25)-\mathrm{Sc}$ & $130.10(14)$ \\
\hline $\mathrm{N}(1)-\mathrm{C}(14)-\mathrm{H}(14)$ & 126.4 & $\mathrm{Si}(2)-\mathrm{C}(25)-\mathrm{H}(25 \mathrm{~A})$ & 104.7 \\
\hline$C(16)-C(15)-C(20)$ & $122.7(3)$ & $\mathrm{Sc}-\mathrm{C}(25)-\mathrm{H}(25 \mathrm{~A})$ & 104.7 \\
\hline $\mathrm{C}(16)-\mathrm{C}(15)-\mathrm{N}(2)$ & $117.9(3)$ & $\mathrm{Si}(2)-\mathrm{C}(25)-\mathrm{H}(25 \mathrm{~B})$ & 104.7 \\
\hline $\mathrm{C}(20)-\mathrm{C}(15)-\mathrm{N}(2)$ & $119.3(3)$ & $\mathrm{Sc}-\mathrm{C}(25)-\mathrm{H}(25 \mathrm{~B})$ & 104.7 \\
\hline$C(15)-C(16)-C(17)$ & $117.2(3)$ & $\mathrm{H}(25 \mathrm{~A})-\mathrm{C}(25)-\mathrm{H}(25 \mathrm{~B})$ & 105.7 \\
\hline$C(15)-C(16)-C(23)$ & $121.7(3)$ & $\mathrm{Si}(1)-\mathrm{C}(26)-\mathrm{H}(26 \mathrm{~A})$ & 109.5 \\
\hline$C(17)-C(16)-C(23)$ & 121.1(3) & $\mathrm{Si}(1)-\mathrm{C}(26)-\mathrm{H}(26 \mathrm{~B})$ & 109.5 \\
\hline$C(18)-C(17)-C(16)$ & $122.3(3)$ & $\mathrm{H}(26 \mathrm{~A})-\mathrm{C}(26)-\mathrm{H}(26 \mathrm{~B})$ & 109.5 \\
\hline $\mathrm{C}(18)-\mathrm{C}(17)-\mathrm{H}(17)$ & 118.8 & $\mathrm{Si}(1)-\mathrm{C}(26)-\mathrm{H}(26 \mathrm{C})$ & 109.5 \\
\hline $\mathrm{C}(16)-\mathrm{C}(17)-\mathrm{H}(17)$ & 118.8 & $\mathrm{H}(26 \mathrm{~A})-\mathrm{C}(26)-\mathrm{H}(26 \mathrm{C})$ & 109.5 \\
\hline$C(17)-C(18)-C(19)$ & $118.4(3)$ & $\mathrm{H}(26 \mathrm{~B})-\mathrm{C}(26)-\mathrm{H}(26 \mathrm{C})$ & 109.5 \\
\hline $\mathrm{C}(17)-\mathrm{C}(18)-\mathrm{C}(22)$ & 121.2(3) & $\mathrm{Si}(1)-\mathrm{C}(27)-\mathrm{H}(27 \mathrm{~A})$ & 109.5 \\
\hline$C(19)-C(18)-C(22)$ & $120.4(3)$ & $\mathrm{Si}(1)-\mathrm{C}(27)-\mathrm{H}(27 \mathrm{~B})$ & 109.5 \\
\hline$C(18)-C(19)-C(20)$ & $122.1(3)$ & $\mathrm{H}(27 \mathrm{~A})-\mathrm{C}(27)-\mathrm{H}(27 \mathrm{~B})$ & 109.5 \\
\hline $\mathrm{C}(18)-\mathrm{C}(19)-\mathrm{H}(19)$ & 118.9 & $\mathrm{Si}(1)-\mathrm{C}(27)-\mathrm{H}(27 \mathrm{C})$ & 109.5 \\
\hline
\end{tabular}




$\begin{array}{llll}\text { H(27A)-C(27)-H(27C) } & 109.5 & \mathrm{H}(29 \mathrm{~B})-\mathrm{C}(29)-\mathrm{H}(29 \mathrm{C}) & 109.5 \\ \text { H(27B)-C(27)-H(27C) } & 109.5 & \mathrm{Si}(2)-\mathrm{C}(30)-\mathrm{H}(30 \mathrm{~B}) & 109.5 \\ \mathrm{Si}(1)-\mathrm{C}(28)-\mathrm{H}(28 \mathrm{~A}) & 109.5 & \mathrm{H}(29 \mathrm{~A})-\mathrm{C}(29)-\mathrm{H}(29 \mathrm{C}) & 109.5 \\ \mathrm{Si}(1)-\mathrm{C}(28)-\mathrm{H}(28 B) & 109.5 & \mathrm{H}(30 \mathrm{~A})-\mathrm{C}(30)-\mathrm{H}(30 \mathrm{~B}) & 109.5 \\ \text { H(28A)-C(28)-H(28B) } & 109.5 & \mathrm{Si}(2)-\mathrm{C}(30)-\mathrm{H}(30 \mathrm{C}) & 109.5 \\ \mathrm{Si}(1)-\mathrm{C}(28)-\mathrm{H}(28 \mathrm{C}) & 109.5 & \mathrm{H}(30 \mathrm{~A})-\mathrm{C}(30)-\mathrm{H}(30 \mathrm{C}) & 109.5 \\ \mathrm{H}(28 \mathrm{~A})-\mathrm{C}(28)-\mathrm{H}(28 \mathrm{C}) & 109.5 & \mathrm{H}(30 \mathrm{~B})-\mathrm{C}(30)-\mathrm{H}(30 \mathrm{C}) & 109.5 \\ \mathrm{H}(28 \mathrm{~B})-\mathrm{C}(28)-\mathrm{H}(28 \mathrm{C}) & 109.5 & \mathrm{Si}(2)-\mathrm{C}(31)-\mathrm{H}(31 \mathrm{~A}) & 109.5 \\ \mathrm{Si}(2)-\mathrm{C}(29)-\mathrm{H}(29 \mathrm{~A}) & 109.5 & \mathrm{Si}(2)-\mathrm{C}(31)-\mathrm{H}(31 \mathrm{~B}) & 109.5 \\ \mathrm{Si}(2)-\mathrm{C}(29)-\mathrm{H}(29 B) & 109.5 & \mathrm{H}(31 \mathrm{~A})-\mathrm{C}(31)-\mathrm{H}(31 \mathrm{~B}) & 109.5 \\ \mathrm{H}(29 \mathrm{~A})-\mathrm{C}(29)-\mathrm{H}(29 B) & 109.5 & \mathrm{Si}(2)-\mathrm{C}(31)-\mathrm{H}(31 \mathrm{C}) & 109.5 \\ \mathrm{Si}(2)-\mathrm{C}(29)-\mathrm{H}(29 \mathrm{C}) & 109.5 & \mathrm{H}(31 \mathrm{~A})-\mathrm{C}(31)-\mathrm{H}(31 \mathrm{C}) & 109.5 \\ \mathrm{Si}(2)-\mathrm{C}(30)-\mathrm{H}(30 \mathrm{~A}) & 109.5 & \mathrm{H}(31 \mathrm{~B})-\mathrm{C}(31)-\mathrm{H}(31 \mathrm{C}) & 109.5 \\ & & & \end{array}$

